\title{
Convex Hull Representations for Bounded Products of Variables
}

\author{
Kurt M. Anstreicher, Samuel Burer ${ }^{\dagger}$ and Kyungchan Park ${ }^{\ddagger}$
}

April 16, 2020

\begin{abstract}
It is well known that the convex hull of $\{(x, y, x y)\}$, where $(x, y)$ is constrained to lie in a box, is given by the Reformulation-Linearization Technique (RLT) constraints. Belotti et al. (2010) and Miller et al. (2011) showed that if there are additional upper and/or lower bounds on the product $z=x y$, then the convex hull can be represented by adding an infinite family of inequalities, requiring a separation algorithm to implement. Nguyen et al. (2018) derived convex hulls with bounds on $z$ for the more general case of $z=x^{b_{1}} y^{b_{2}}$, where $b_{1} \geq 1, b_{2} \geq 1$. We focus on the most important case where $b_{1}=b_{2}=1$ and show that the convex hull with either an upper bound or lower bound on the product is given by RLT constraints, the bound on $z$ and a single Second-Order Cone (SOC) constraint. With both upper and lower bounds on the product, the convex hull can be represented using no more than three SOC constraints, each applicable on a subset of $(x, y)$ values. In addition to the convex hull characterizations, volumes of the convex hulls with either an upper or lower bound on $z$ are calculated and compared to the relaxation that imposes only the RLT constraints. As an application of these volume results, we show how spatial branching can be applied to the product variable so as to minimize the sum of the volumes for the two resulting subproblems.
\end{abstract}

Keywords: Convex Hull, Second-Order Cone, Bilinear Product, Global Optimization.

\footnotetext{
*Department of Business Analytics, Tippie College of Business, University of Iowa, Iowa City, IA 52242. kurt-anstreicher@uiowa.edu

${ }^{\dagger}$ Department of Business Analytics, Tippie College of Business, University of Iowa, Iowa City, IA 52242. samuel-burer@uiowa.edu

${ }^{\ddagger}$ Department of Business Analytics, Tippie College of Business, University of Iowa, Iowa City, IA 52242. kyungchan-park@uiowa.edu
} 


\section{Introduction}

Representing the product of two variables is a fundamental problem in global optimization. This issue arises naturally in the presence of bilinear terms in the objective and/or constraints, and also when more complex functions are decomposed into factorable form by global optimization algorithms such as BARON [13]. It is well known [1] that the convex hull of $(x, y, x y)$ where $(x, y)$ lie in a box is given by the four Reformulation-Linearization Technique (RLT) constraints [15, 16], also often referred to as the McCormick inequalities. Linderoth [10] derived the convex hulls of bilinear functions over triangles and showed that they have Second-Order Cone (SOC) [6] representations. Dey et al. [7] show that the convex hull of $(x, y, x y)$ over the box intersected with a bilinear equation is SOC representable. The convex hull for the complete 5-variable quadratic system that arises from 2 original variables in a box was considered in [3] and [8]. Explicit functional forms for the convex hull that apply over a dissection of the box are given in [8], while [3] shows that the convex hull can be represented using the RLT constraints and a PSD condition.

The focus of this paper is to consider the convex hull of $(x, y, x y)$ when $(x, y)$ lie in a box and there are explicit upper and/or lower bounds on the product $x y$. More precisely, we wish to characterize the convex hull of

$$
\mathcal{F}^{\prime}:=\left\{(x, y, z): z=x y, l_{x} \leq x \leq u_{x}, l_{y} \leq y \leq u_{y}, l_{z} \leq z \leq u_{z}\right\}
$$

where $0 \leq\left(l_{x}, l_{y}, l_{z}\right)<\left(u_{x}, u_{y}, u_{z}\right)$. We assume $\mathcal{F}^{\prime} \neq \emptyset$, i.e., that $l_{x} l_{y} \leq l_{z}<u_{z} \leq u_{x} u_{y}$. When $l_{x} l_{y}<l_{z}$, we say that the lower bound $l_{z}$ on $z$ is non-trivial and similarly for the upper bound when $u_{z}<u_{x} u_{y}$. By a simple rescaling, we can transform the feasible region to have $u_{x}=u_{y}=1$, and we will make this assumption throughout. Note also that if $z=x y$ and $l_{y}>0$ then $x \leq u_{z} / l_{y}$, so we could assume that $u_{x} \leq u_{z} / l_{y}$. Then $u_{x}=1$ means we can assume $l_{y} \leq u_{z}$, and similarly $l_{x} \leq u_{z}$. In addition $x \geq l_{z} / u_{y}$, so $u_{y}=1$ implies that we may assume that $l_{x} \geq l_{z}$ and similarly $l_{y} \geq l_{z}$. Combining these facts, we could assume that

$$
l_{z} \leq l_{x} \leq u_{z}, \quad l_{z} \leq l_{y} \leq u_{z} .
$$


Said differently, if $l_{x}$ and $l_{y}$ do not satisfy (1), we can adjust them so that they do. However, we do not explicitly assume that (1) holds until Section 4.

The problem of characterizing $\operatorname{conv}\left(\mathcal{F}^{\prime}\right)$ has been considered in several previous works. Bellotti et.al. [5] and Miller et.al. [11] show that $\operatorname{conv}\left(\mathcal{F}^{\prime}\right)$ can be represented by the RLT inequalities, bounds on $z$ and lifted tangent inequalities, which we describe in Section 2. Since the lifted tangent inequalities belong to an infinite family, they require a separation algorithm to implement. The convex hull for a generalization of $\mathcal{F}^{\prime}$ where $z=x^{b_{1}} y^{b_{2}}, b_{1} \geq 1, b_{2} \geq 1$ is considered in [12]. There are two primary differences between this paper and [12]. First, because [12] considers a more general problem, both the analysis required and the representations obtained are substantially more complex than our results here. In particular, we will show that with $b_{1}=b_{2}=1$, the convex hull of $\mathcal{F}^{\prime}$ can always be represented using linear inequalities and SOC constraints, although in some cases the derivations of the SOC forms for these constraints is nontrivial. A second difference is that [12] assumes $l_{x}>0, l_{y}>0$. In [12] it is stated that this assumption is without loss of generality, since by a limiting argument positive lower bounds could be reduced to zero. This is true, but [12] goes on to assume that $l_{x}=l_{y}=1$, making representations for the important case of $l_{x}=0$ and/or $l_{y}=0$ difficult to extract from the results. Another recent, related paper by Santana and Dey [14] shows that $\operatorname{conv}\left(\mathcal{F}^{\prime}\right)$ is SOC representable using a disjunctive representation in a lifted space; see section 4 therein. In contrast, we will show that $\operatorname{conv}\left(\mathcal{F}^{\prime}\right)$ is SOC representable directly in the variables $(x, y, z)$.

In Section 3, we consider the case where $l_{x}=l_{y}=0$ and there are non-trivial upper and/or lower bounds on the product variable $z$. Our methodology for obtaining explicit representations for $\operatorname{conv}\left(\mathcal{F}^{\prime}\right)$ is based on the lifted tangent inequalities of [5, 11]. We do not use the inequalities per se, but rather show how the process by which they are constructed can be re-interpreted to generate nonlinear inequalities. We show that in all cases these inequalities can be put into the form of SOC constraints, so that $\operatorname{conv}\left(\mathcal{F}^{\prime}\right)$ is SOC-representable [6]. In the presence of both non-trivial upper and lower bounds on $z$, the representation requires a dissection of the domain of $(x, y)$ values into three regions, each of which uses a different SOC constraint to obtain the convex hull. One of the three SOC constraints is globally valid, and the use of this one constraint 
together with the RLT constraints and bounds on $z$ empirically gives a close approximation of $\operatorname{conv}\left(\mathcal{F}^{\prime}\right)$. Finally we compute the volumes of $\operatorname{conv}\left(\mathcal{F}^{\prime}\right)$ as given in the case where there is either a non-trivial upper or non-trivial lower bound on $z$ using an SOC constraint, the RLT constraints and bound on $z$, and compare these volumes to the volumes of the regions where the SOC constraint is omitted. This comparison is similar to the volume computations in [2], where the effect of adding a PSD condition to the RLT constraints was considered. An interesting application of these computations is to consider the reduction in volume associated with spatial branching [4] based on the product variable $z$.

In Section 4 we generalize the results of Section 3 to consider positive lower bounds on $(x, y)$, specifically bounds $l_{x} \geq 0, l_{y} \geq 0$. We again show that in all cases $\operatorname{conv}\left(\mathcal{F}^{\prime}\right)$ is $\operatorname{SOC}$ representable. As in the case of $l_{x}=l_{y}=0$, when there is a non-trival upper or a non-trivial lower bound on the product, but not both, the representation of $\operatorname{conv}\left(\mathcal{F}^{\prime}\right)$ requires only a single SOC constraint in addition to the RLT constraints and bound on $z$. When there are both nontrivial lower and upper bounds on $z$ there are several cases to consider, again requiring up to three SOC constraints, each applicable on a subset of the domain of $(x, y)$. We close the paper in Section 5 with a summary of the results and some promising directions for future research.

\section{Lifted Tangent Inequalities}

The set $\mathcal{F}=\left\{(x, y, z): z=x y, l_{x} \leq x \leq u_{x}, l_{y} \leq y \leq u_{y}\right\}$, i.e., $\mathcal{F}^{\prime}$ with only trivial bounds on $z$, is not convex, but it is well known that $\operatorname{conv}(\mathcal{F})$ is the linear envelope of four extreme points [1]. This linear envelope can be given by the four RLT constraints [16]:

$$
\begin{aligned}
& z \geq u_{y} x+u_{x} y-u_{x} u_{y}, \\
& z \geq l_{y} x+l_{x} y-l_{x} l_{y}, \\
& z \leq u_{y} x+l_{x} y-l_{x} u_{y} \\
& z \leq l_{y} x+u_{x} y-u_{x} l_{y} .
\end{aligned}
$$

Figure 1 shows the product $x y$ as a colored surface and the boundary edges for the linear envelope as red lines for the case where $l_{x}=l_{y}=0$ and $u_{x}=u_{y}=1$. 


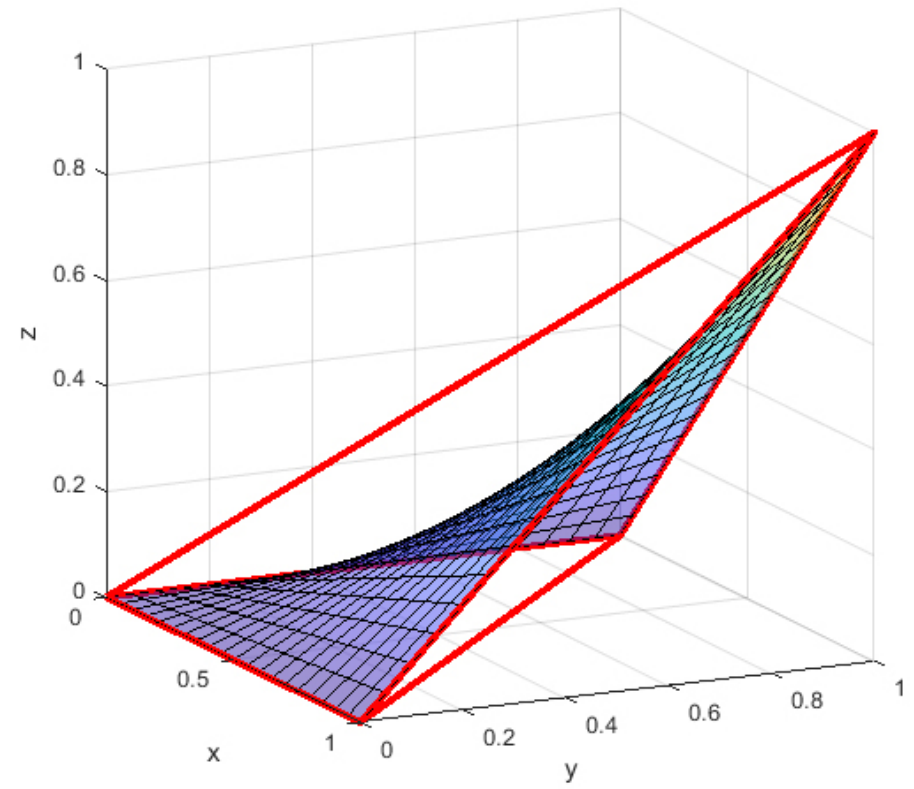

Figure 1: Convex hull with no bounds on $z$

The focus in this paper is to represent the convex hull of the set $\mathcal{F}^{\prime}$, corresponding to $\mathcal{F}$ with nontrivial upper and lower bounds on the product variable $z$. Recall that we assume $u_{x}=$ $u_{y}=1$ throughout. It is shown in [5, 11] that the convex hull of $\mathcal{F}^{\prime}$ is given by the RLT constraints, bounds on $z$, and lifted tangent inequalities. Our technique for deriving convex hull representations in Sections 3-4 is based on the construction of these lifted tangent inequalities, which we now describe. Assume that $l=\left(l_{x}, l_{y}, l_{z}\right)$ and $u=\left(1,1, u_{z}\right)$. The construction of a lifted tangent inequality based on a point $\left(x^{*}, y^{*}, l_{z}\right) \in \mathcal{F}^{\prime}$ proceeds as follows. The inequality tangent to the curve $x y=l_{z}$ at $\left(x^{*}, y^{*}\right)$ has the form

$$
y^{*}\left(x-x^{*}\right)+x^{*}\left(y-y^{*}\right) \geq 0 .
$$

This inequality is lifted to an inequality in the variables $(x, y, z)$ of the form

$$
y^{*}\left(x-x^{*}\right)+x^{*}\left(y-y^{*}\right)+a\left(z-l_{z}\right) \geq 0,
$$

with $a<0$. The value of $a$ is chosen so that there is a point $\left(\bar{x}, \bar{y}, u_{z}\right) \in \mathcal{F}^{\prime}$ such that the inequality (3) is tight at $\left(\bar{x}, \bar{y}, u_{z}\right)$, and (3) is valid for $\mathcal{F}^{\prime}$. There are two possibilities for such a point: 
- $\bar{x}=\rho x^{*}, \bar{y}=\rho y^{*}$, where $\rho=\sqrt{u_{z} / l_{z}}$. In this case the value of $a$ is independent of $\left(x^{*}, y^{*}\right)$; there is an expression for $a$ that depends only on $l_{z}$ and $u_{z}[5,11]$.

- $(\bar{x}, \bar{y})$ corresponds to one of the endpoints of the curve $x y=u_{z}$ for the given bounds on $x$ and $y$. In the case of $u_{z}=1$, this point is $\bar{x}=\bar{y}=1$.

The construction of a lifted tangent inequality can alternatively start with a point $\left(\bar{x}, \bar{y}, u_{z}\right) \in$ $\mathcal{F}^{\prime}$. In this case the roles of $\left(x^{*}, y^{*}\right)$ and $(\bar{x}, \bar{y})$ are reversed, and either $\left(x^{*}, y^{*}\right)=(1 / \rho)(\bar{x}, \bar{y})$ or $\left(x^{*}, y^{*}\right)$ is an endpoint of the curve $x y=l_{z}$ for the bounds on $x$ and $y$. If $l_{x} l_{y}=l_{z}$ then this point is $\left(l_{x}, l_{y}, l_{z}\right)$; for example if $l_{x}=l_{y}=l_{z}=0$, the point is $(0,0,0)$.

In all cases the result of the above process is an inequality that is valid for $\mathcal{F}^{\prime}$, and which is tight for a line segment joining two points $\left(x^{*}, y^{*}, l_{z}\right) \in \mathcal{F}^{\prime}$ and $\left(\bar{x}, \bar{y}, u_{z}\right) \in \mathcal{F}^{\prime}$. Our approach does not use the lifted tangent inequalities themselves but is rather based on the process for constructing them. In particular, starting with a point $(x, y)$ with $x \in\left[l_{x}, 1\right], y \in\left[l_{y}, 1\right], l_{z}<$ $x y<u_{z}$, we determine the two points $\left(x^{*}, y^{*}, l_{z}\right) \in \mathcal{F}^{\prime}$ and $\left(\bar{x}, \bar{y}, u_{z}\right) \in \mathcal{F}^{\prime}$ so that the lifted tangent inequality that is tight at $(x, y, z)$ is tight for the line segment joining $\left(x^{*}, y^{*}, l_{z}\right)$ and $\left(\bar{x}, \bar{y}, u_{z}\right)$. Suppose that $0 \leq \alpha \leq 1$ is such that $(x, y)=\alpha\left(x^{*}, y^{*}\right)+(1-\alpha)(\bar{x}, \bar{y})$. Then the constraint $z \leq \alpha l_{z}+(1-\alpha) u_{z}$ is valid and tight on the line segment between $\left(x^{*}, y^{*}, l_{z}\right)$ and $\left(\bar{x}, \bar{y}, u_{z}\right)$. If $\alpha$ can be expressed as a function of $(x, y)$ then the result is a single nonlinear constraint that is equivalent to a family of lifted tangent inequalities. Our goal will be to obtain such a constraint and show that it can always be expressed in the form of an SOC constraint. An SOC constraint obtained in this manner is certainly valid over the $\{(x, y)\}$ domain on which it is derived, since it is equivalent to the lifted tangent inequalities on that domain. In some cases we obtain SOC constraints that are actually globally valid, that is, valid for all $(x, y, z) \in \operatorname{conv}\left(\mathcal{F}^{\prime}\right)$.

\section{Convex hull representation with $l_{x}=l_{y}=0$}

In this section we obtain representations for $\operatorname{conv}\left(\mathcal{F}^{\prime}\right)$ when $l_{x}=l_{y}=0$ and $u_{x}=u_{y}=1$. We begin by considering the case where $l_{z}=0, u_{z}<1$, and next consider the case where $l_{z}>0$, $u_{z}=1$. In both of these cases we show that a combination of the RLT constraints, the bound 
on $z$, and a single SOC constraint gives the convex hull of $\mathcal{F}^{\prime}$. In the case where $l_{z}>0$ and $u_{z}<1$ we show that the convex hull of $\mathcal{F}^{\prime}$ is representable using three SOC constraints, each applicable on a subset of the domain in $(x, y)$. One of these SOC constraints is globally valid, and the combination of that single SOC constraint, the RLT constraints and the bounds on $z$ empirically gives a close approximation of $\operatorname{conv}\left(\mathcal{F}^{\prime}\right)$.

\section{$3.1 \quad$ Non-trivial upper bound on $x y$ with $l_{x}=l_{y}=0$}

We first consider the case where $x \in[0,1], y \in[0,1]$ and we impose a non-trivial upper bound on the product $z \leq u_{z}$.

Proposition 1. Let $l=(0,0,0), u=\left(1,1, u_{z}\right)$ where $0<u_{z}<1$. Then $\operatorname{conv}\left(\mathcal{F}^{\prime}\right)$ is given by the RLT constraints (2), the bound $z \leq u_{z}$ and the SOC constraint $z^{2} \leq u_{z} x y$.

Proof. From [5, 11] the convex hull of $\mathcal{F}^{\prime}$ is given by the RLT constraints, bounds on $z$ and the lifted tangent inequalities. In this case each lifted tangent inequality is obtained by taking a point $(\bar{x}, \bar{y})=\left(t, u_{z} / t\right)$ with $u_{z} \leq t \leq 1$, forming the tangent equation to $x y=u_{z}$ at $\left(t, u_{z} / t\right)$, and lifting this tangent equation to form a valid inequality of the form

$$
\frac{u_{z}}{t} x+t y-2 z \geq 0
$$

The set of points in $\mathcal{F}^{\prime}$ that satisfy (4) with equality then consists of the line segment joining the points $\left(t, u_{z} / t, u_{z}\right)$ and $(0,0,0)$. The constraint $z^{2} \leq u_{z} x y$ holds with equality for all such points, and therefore implies all of the lifted tangent inequalities. Moreover, $z^{2} \leq u_{z} x y$ clearly holds for all $(x, y, z) \in \operatorname{conv}\left(\mathcal{F}^{\prime}\right)$, since if $z=x y \leq u_{z}$ then $z^{2}=(x y)^{2} \leq u_{z} x y$.

In Figure 2, we illustrate $\operatorname{conv}\left(\mathcal{F}^{\prime}\right)$ for the case of $l=(0,0,0), u=(1,1,0.4)$. The green surface illustrates the boundary of the SOC cone, and the red solid lines indicate edges on the boundary of $\operatorname{conv}\left(\mathcal{F}^{\prime}\right)$ corresponding to the RLT constraints and the upper bound $z \leq u_{z}$. The dashed red lines indicate edges of the polyhedron corresponding to the RLT constraints and upper bound on $z$, highlighting the portion cut away by the SOC constraint. Note that none of the RLT constraints are redundant, and although the SOC constraint is globally valid, 


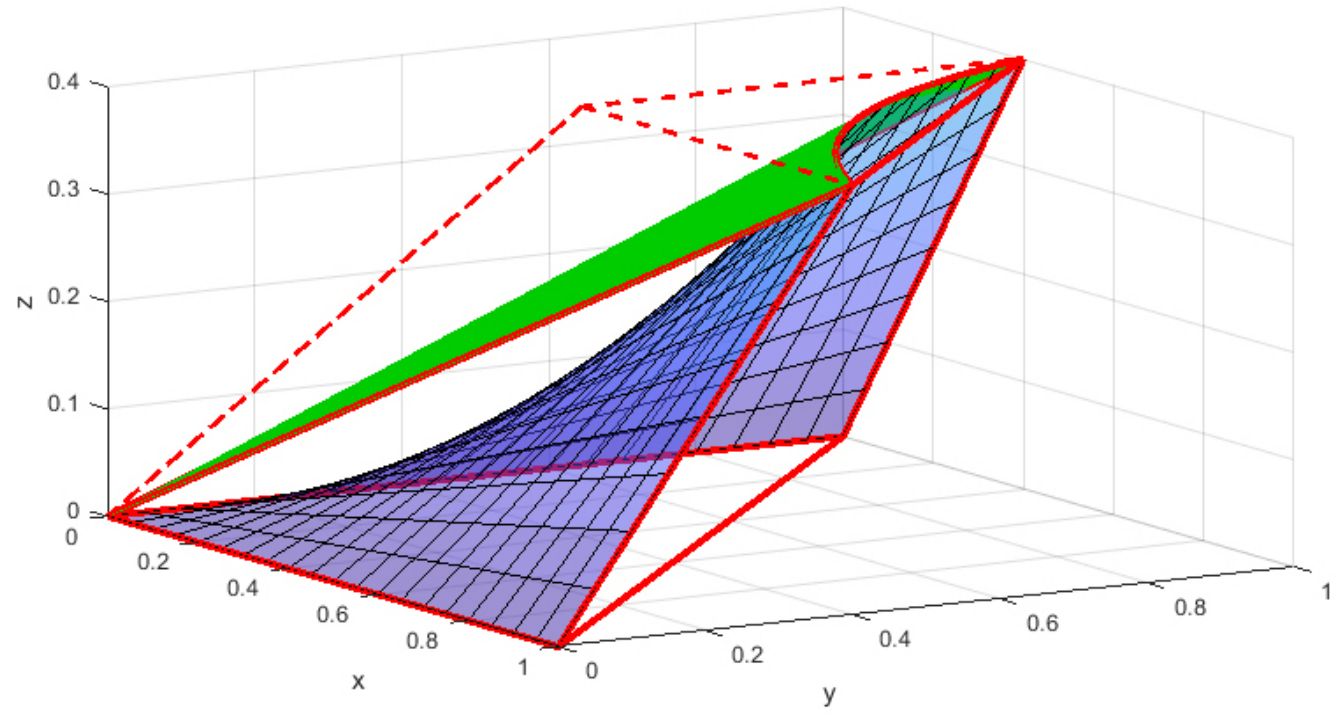

Figure 2: Convex hull with upper bound on $z$

this constraint does not give a tight upper bound on $z$ for all feasible $(x, y)$, unlike the case considered in Section 3.2. In [5], it is noted that if $l=\left(0,0, l_{z}\right)$ and $u=\left(+\infty,+\infty, u_{z}\right)$ then $\operatorname{conv}\left(\mathcal{F}^{\prime}\right)$ is given by the bounds on $z$ and the SOC constraint $\left(z+\sqrt{l_{z} u_{z}}\right)^{2} \leq\left(\sqrt{l_{z}}+\sqrt{u_{z}}\right)^{2} x y$; when $l_{z}=0$ this is exactly the constraint $z^{2} \leq u_{z} x y$.

\subsection{Non-trivial lower bound on $x y$ with $l_{x}=l_{y}=0$}

We next consider the case where $x \in[0,1], y \in[0,1]$ and we impose only a lower bound on the product $z=x y \geq l_{z}$. To obtain an SOC representation for $\operatorname{conv}\left(\mathcal{F}^{\prime}\right)$ we need to characterize the lifted tangent inequalities, as in the proof of Proposition 1. This is more complex than for the case of an upper bound $z \leq u_{z}$ because the lifted tangent inequalities are now tight on line segments of the form $\alpha\left(\bar{x}, \bar{y}, l_{z}\right)+(1-\alpha)(1,1,1)$, where $\bar{x} \bar{y}=l_{z}$.

Proposition 2. Let $l=\left(0,0, l_{z}\right), u=(1,1,1)$ where $0<l_{z}<1$. Then $\operatorname{conv}\left(\mathcal{F}^{\prime}\right)$ is given by the RLT constraint (2a), the bound $z \geq l_{z}$ and the SOC constraint $\sqrt{(\hat{x}, \hat{y}) M(\hat{x}, \hat{y})^{T}} \leq x+y-2 z$ where $\hat{x}:=1-x, \hat{y}:=1-y$, and

$$
M=\left(\begin{array}{cc}
1 & 2 l_{z}-1 \\
2 l_{z}-1 & 1
\end{array}\right) \succeq 0 .
$$


Proof. Given a point $(x, y)$ with $x>l_{z}, y>l_{z}$ and $x y>l_{z}$, a lifted tangent inequality that is tight at $(x, y, z)$ must have $x=\alpha x^{*}+(1-\alpha), y=\alpha y^{*}+(1-\alpha)$. Writing $x^{*}$ and $y^{*}$ in terms of $x, y$ and $\alpha$ and using $x^{*} y^{*}=l_{z}$ results in a quadratic equation for $\alpha$. Substituting the appropriate root of this quadratic equation into the constraint $z \leq \alpha l_{z}+(1-\alpha)$ then obtains the equivalent inequality

$$
z \leq \frac{(x+y)-\sqrt{(x-y)^{2}+4 l_{z}(1-x)(1-y)}}{2} .
$$

It is straightforward to verify that

$$
\left(\begin{array}{l}
1-x \\
1-y
\end{array}\right)^{T}\left(\begin{array}{cc}
1 & 2 l_{z}-1 \\
2 l_{z}-1 & 1
\end{array}\right)\left(\begin{array}{l}
1-x \\
1-y
\end{array}\right)=(x-y)^{2}+4 l_{z}(1-x)(1-y) .
$$

The constraint $\sqrt{(\hat{x}, \hat{y}) M(\hat{x}, \hat{y})^{T}} \leq x+y-2 z$ then implies all of the lifted tangent inequalities, and $0 \leq l_{z} \leq 1$ implies that $-1 \leq 1-2 l_{z} \leq 1$, so $M \succeq 0$. Therefore the convex hull of $\mathcal{F}^{\prime}$ is given by the RLT constraints, the bound $z \geq l_{z}$ and this one SOC constraint. However the RLT constraints (2b)-2d are easily shown to be redundant, even if $l_{x}$ and $l_{y}$ are increased to $l_{z}$ in their definitions.

In Figure 3, we illustrate $\operatorname{conv}\left(\mathcal{F}^{\prime}\right)$ for the case of $l=(0,0,0.2), u=(1,1,1)$. In the figure the dashed lines indicate edges corresponding to the RLT constraints $(2 \mathrm{c})-(2 \mathrm{~d})$, with $l_{x}$ and $l_{y}$ increased to $l_{z}=0.2$ in the formulas for these constraints, as in (1). Note that in this case the SOC constraint gives a tight upper bound on $z$ for all feasible $(x, y)$, unlike the case illustrated in Figure 2.

\subsection{Non-trivial lower and upper bounds on $x y$ with $l_{x}=l_{y}=0$}

We now consider the case where both non-trivial lower and upper bounds are imposed on the product $z=x y$, so $0<l_{z}<u_{z}<1$. The situation becomes more complex than with only an upper or lower bound because now there are 3 classes of lifted tangent inequalities. In each of the cases below, $\left(x^{*}, y^{*}, l_{z}\right) \in \mathcal{F}^{\prime}$.

1. For the "center" domain $y \geq u_{z} x$ and $x \geq u_{z} y$, each lifted tangent inequality corresponds to a line segment connecting $\left(x^{*}, y^{*}, l_{z}\right)$ and $\left(\bar{x}, \bar{y}, u_{z}\right) \in \mathcal{F}^{\prime}$, where $\bar{x} \bar{y}=u_{z},(\bar{x}, \bar{y})=$ $\rho\left(x^{*}, y^{*}\right)$ and $\rho=\sqrt{u_{z} / l_{z}}$. 


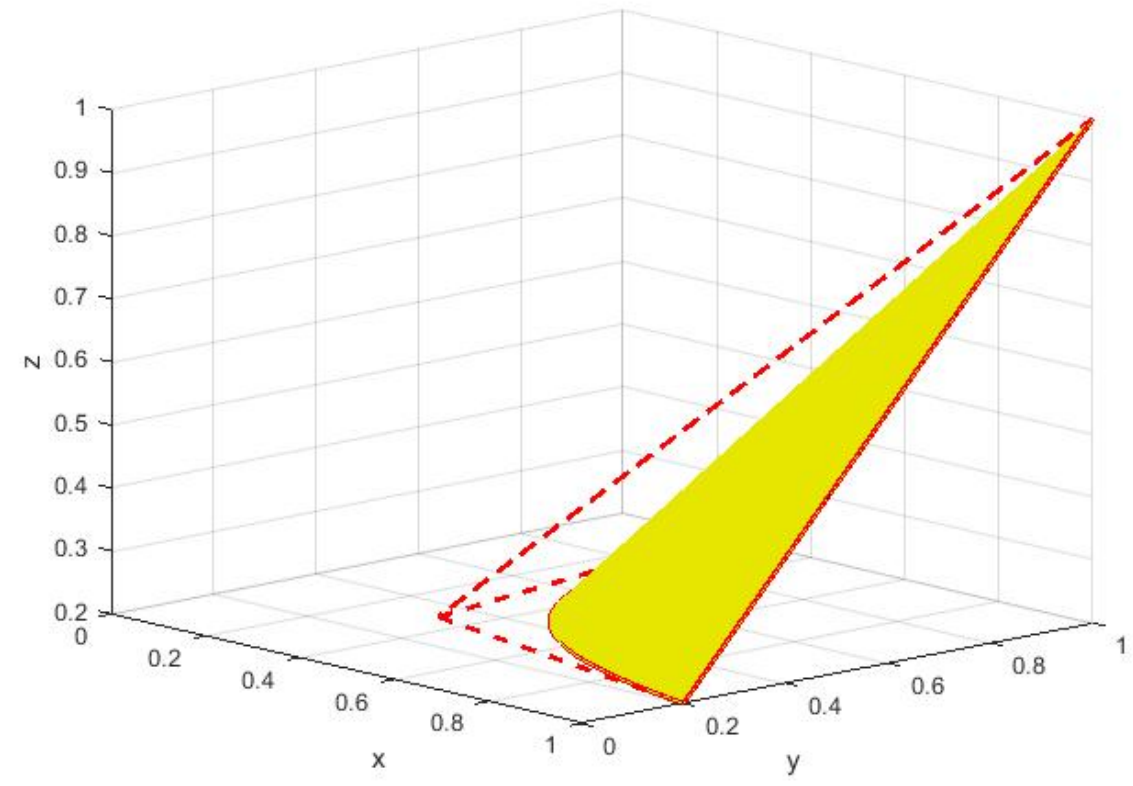

Figure 3: Convex hull with lower bound on $z$

2. For the "side" domain $y \leq u_{z} x$, each lifted tangent inequality corresponds to a line segment connecting $\left(x^{*}, y^{*}, l_{z}\right)$ and $\left(1, u_{z}, u_{z}\right)$.

3. For the "side" domain $x \leq u_{z} y$, each lifted tangent inequality corresponds to a line segment connecting $\left(x^{*}, y^{*}, l_{z}\right)$ and $\left(u_{z}, 1, u_{z}\right)$.

Figure 4 depicts these three domains in the $x y$-space for the case where $l_{z}=0.2, u_{z}=0.7$.

In the lemma below, we show that in this $\operatorname{case} \operatorname{conv}\left(\mathcal{F}^{\prime}\right)$ can be represented using a single RLT constraint, the bounds on $z$, and 3 different SOC constraints, each applicable on one of the domains described above. For convenience in stating the result, we define matrices

$$
M_{1}:=\left(\begin{array}{cc}
u_{z}{ }^{2} & 2 l_{z}-u_{z} \\
2 l_{z}-u_{z} & 1
\end{array}\right), \quad M_{2}:=\left(\begin{array}{cc}
1 & 2 l_{z}-u_{z} \\
2 l_{z}-u_{z} & u_{z}{ }^{2}
\end{array}\right) .
$$

Proposition 3. Let $l=\left(0,0, l_{z}\right), u=\left(1,1, u_{z}\right)$ where $0<l_{z}<u_{z}<1$. Then $\operatorname{conv}\left(\mathcal{F}^{\prime}\right)$ is given by the RLT constraint (2a), the bounds $l_{z} \leq z \leq u_{z}$ and three SOC constraints, each applicable in a different region:

1. The constraint $\left(z+\sqrt{l_{z} u_{z}}\right)^{2} \leq\left(\sqrt{l_{z}}+\sqrt{u_{z}}\right)^{2} x y$, applicable if $y \geq u_{z} x$ and $x \geq u_{z} y$.

2. The constraint $\sqrt{(\hat{x}, \hat{y}) M_{1}(\hat{x}, \hat{y})^{T}} \leq u_{z} x+y-2 z$, where $\hat{x}:=1-x, \hat{y}:=u_{z}-y$ and $M_{1} \succeq 0$ is given in (5), applicable if $y \leq u_{z} x$. 


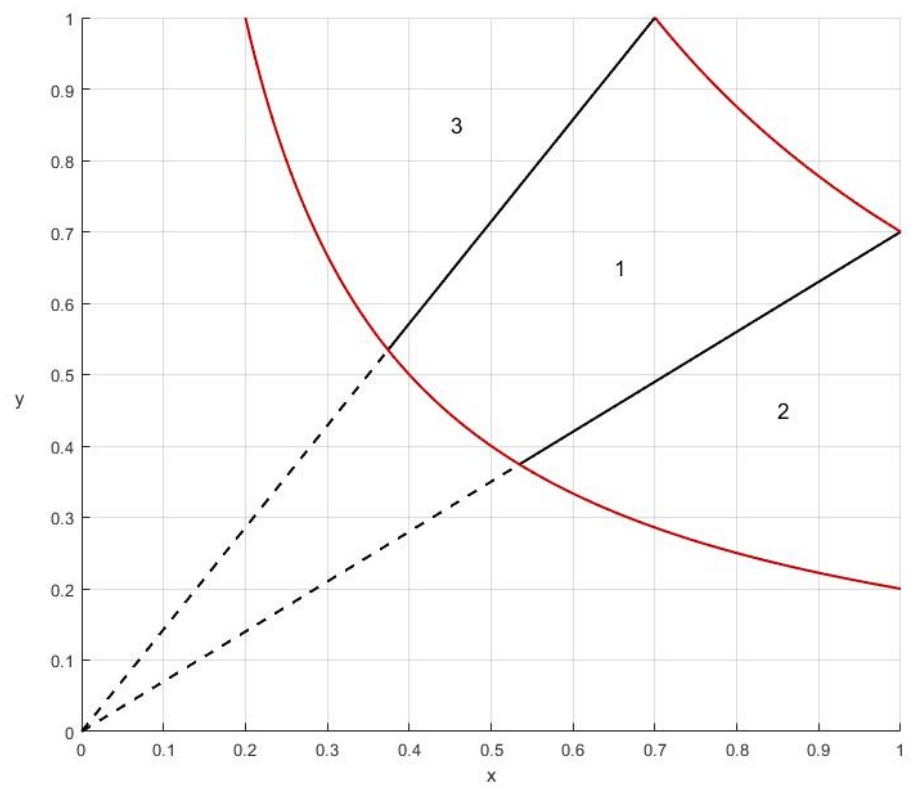

Figure 4: Domains for SOC constraints with lower and upper bounds on $z$

3. The constraint $\sqrt{(\hat{x}, \hat{y}) M_{2}(\hat{x}, \hat{y})^{T}} \leq x+u_{z} y-2 z$, where $\hat{x}:=u_{z}-x, \hat{y}:=1-y$ and $M_{2} \succeq 0$ is given in (5), applicable if $x \leq u_{z} y$.

Proof. Assume first that $y \geq u_{z} x$ and $x \geq u_{z} y$. We know that $(x, y)$ is on the line segment connecting $\left(x^{*}, y^{*}\right)$ and $(\bar{x}, \bar{y})$, from which we conclude that $x^{*}=x \sqrt{l_{z} /(x y)}$ and $\bar{x}=x \sqrt{u_{z} /(x y)}$. Then $x=\alpha x^{*}+(1-\alpha) \bar{x}$ implies that

$$
x=\alpha x \sqrt{l_{z} /(x y)}+(1-\alpha) x \sqrt{u_{z} /(x y)}
$$

from which we obtain $\sqrt{x y}=\alpha \sqrt{l_{z}}+(1-\alpha) \sqrt{u_{z}}$, or

$$
\alpha=\frac{\sqrt{u_{z}}-\sqrt{x y}}{\sqrt{u_{z}}-\sqrt{l_{z}}} .
$$

Substituting this value of $\alpha$ into the inequality $z \leq \alpha l_{z}+(1-\alpha) u_{z}$ and simplifying, we obtain the inequality $\left(z+\sqrt{l_{z} u_{z}}\right)^{2} \leq\left(\sqrt{l_{z}}+\sqrt{u_{z}}\right)^{2} x y$. Therefore, this SOC constraint implies all of the lifted tangent inequalities if $y \geq u_{z} x$ and $x \geq u_{z} y$.

Next assume that $y \leq u_{z} x$. The situation is now very similar to that encountered in the proof of Proposition 2, except that the lifted tangent inequality is tight on a line segment connecting a 
point $\left(x^{*}, y^{*}, l_{z}\right)$ with $x^{*} y^{*}=l_{z}$ to the point $\left(1, u_{z}, u_{z}\right)$, rather than $(1,1,1)$. A similar process to that used in the proof of Proposition 2 again results in a quadratic equation for $\alpha$ such that $x=\alpha x^{*}+(1-\alpha), y=\alpha y^{*}+(1-\alpha) u_{z}$, and substituting the appropriate root into the inequality $z \leq \alpha l_{z}+(1-\alpha) u_{z}$ results in the inequality

$$
z \leq \frac{u_{z} x+y-\sqrt{\left(u_{z} x-y\right)^{2}+4 l_{z}(1-x)\left(u_{z}-y\right)}}{2} .
$$

It is straightforward to verify that $\left(u_{z} x-y\right)^{2}+4 l_{z}(1-x)\left(u_{z}-y\right)=(\hat{x}, \hat{y}) M_{1}(\hat{x}, \hat{y})^{T}$, where $\hat{x}:=1-x, \hat{y}:=u_{z}-y$, and $M_{1} \succeq 0$ follows from $l_{z} \leq u_{z}$. Therefore, the constraint $\sqrt{(\hat{x}, \hat{y}) M_{1}(\hat{x}, \hat{y})^{T}} \leq u_{z} x+y-2 z$ implies the lifted tangent inequalities when $y \leq u_{z} x$. The analysis when $x \leq u_{z} y$ is very similar, interchanging the roles of $x$ and $y$.

Note that if $u_{z}=1$ then $M_{1}=M_{2}=M$, where $M \succeq 0$ was given in Proposition 2. In this case we always have either $x \leq y$ or $y \leq x$, so the "center" SOC constraint is not present and the two "side" SOC constraints are identical and equal to the constraint in Proposition 2. If $l_{z}=0$, then the SOC constraint that applies when $y \geq u_{z} x$ and $x \geq u_{z} y$ is identical to the SOC constraint from Proposition 1. Moreover, if $l_{z}=0$ and $y \leq u_{z} x$, then $(\hat{x}, \hat{y}) M_{1}(\hat{x}, \hat{y})^{T}=\left(u_{z} x-\right.$ $y)^{2}$, and the SOC constraint $\sqrt{(\hat{x}, \hat{y}) M_{1}(\hat{x}, \hat{y})^{T}} \leq u_{z} x+y-2 z$ is exactly the RLT constraint $z \leq y$. Similarly for $l_{z}=0$ and $x \leq u_{z} y$, the SOC constraint $\sqrt{(\hat{x}, \hat{y}) M_{2}(\hat{x}, \hat{y})^{T}} \leq x+u_{z} y-2 z$ becomes the RLT constraint $z \leq x$.

In Figure 5, we illustrate $\operatorname{conv}\left(\mathcal{F}^{\prime}\right)$ for $l=(0,0,0.2), u=(1,1,0.7)$. As in Figure 3 the dashed red lines indicate edges corresponding to the RLT constraints $2 \mathrm{c}-(2 \mathrm{~d})$, with $l_{x}$ and $l_{y}$ increased to $l_{z}=0.2$ in the formulas for these constraints, as in (1).

It is easy to show that the "side" SOC constraints from Proposition 3 that are applicable on the domains $y \leq u_{z} x$ and $x \leq u_{z} y$ are not valid outside these domains. However the "center" constraint is valid for all $(x, y, z) \in \operatorname{conv}\left(\mathcal{F}^{\prime}\right)$. To see this, note that if $l_{z} \leq z=x y \leq u_{z}$, then

$$
\begin{aligned}
\left(\sqrt{u_{z}}-\sqrt{x y}\right)\left(\sqrt{x y}-\sqrt{l_{z}}\right) & \geq 0 \\
\left(\sqrt{l_{z}}+\sqrt{u_{z}}\right) \sqrt{x y} & \geq x y+\sqrt{l_{z} u_{z}} \\
\left(z+\sqrt{l_{z} u_{z}}\right)^{2} & \leq\left(\sqrt{l_{z}}+\sqrt{u_{z}}\right)^{2} x y .
\end{aligned}
$$




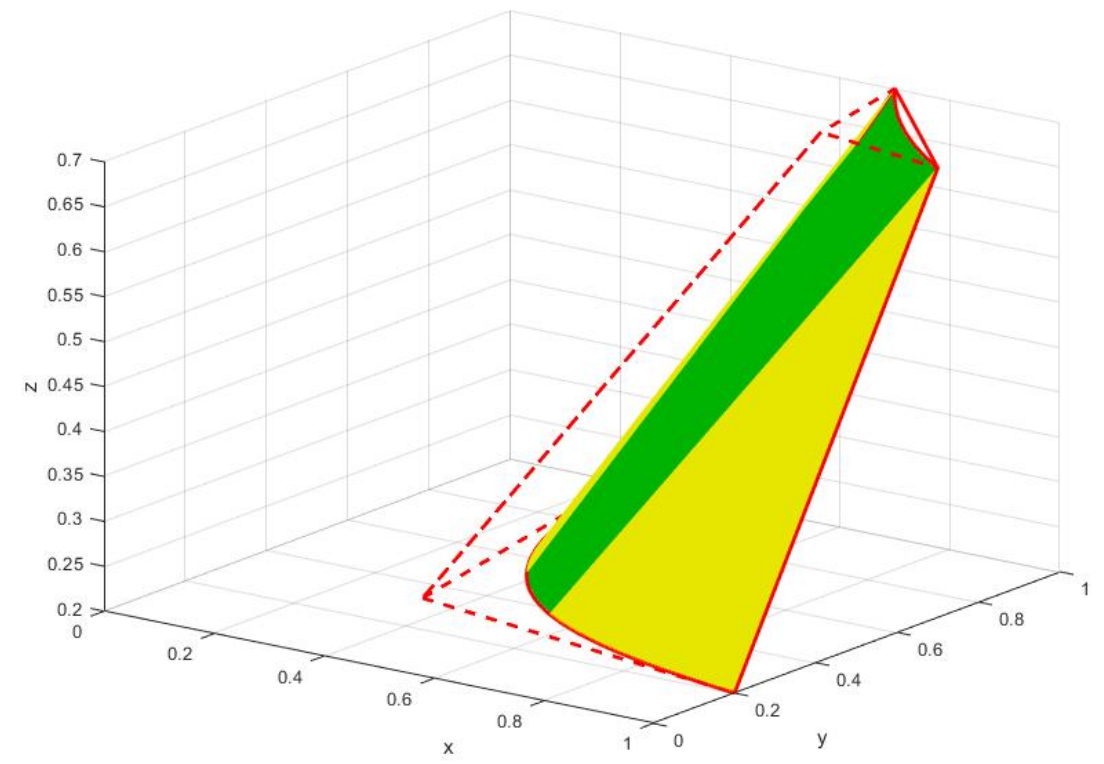

Figure 5: Convex hull with lower and upper bounds on $z$

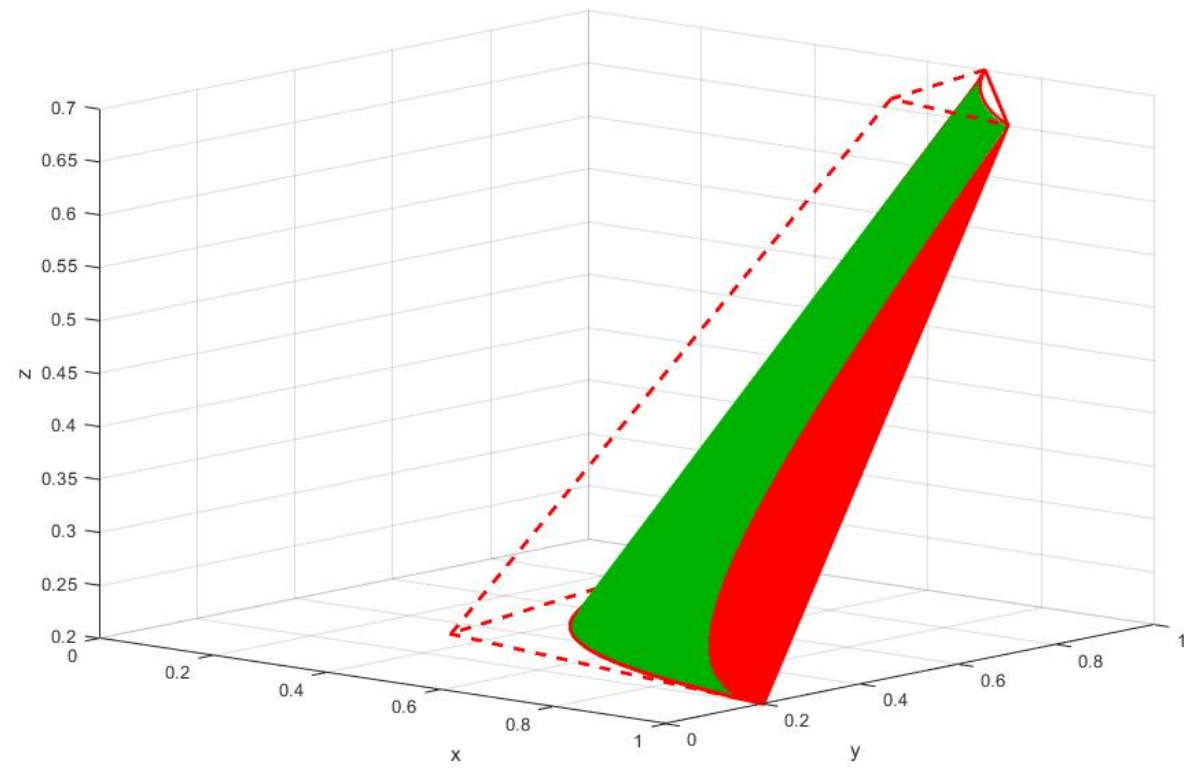

Figure 6: Center cone only with RLT constraints 


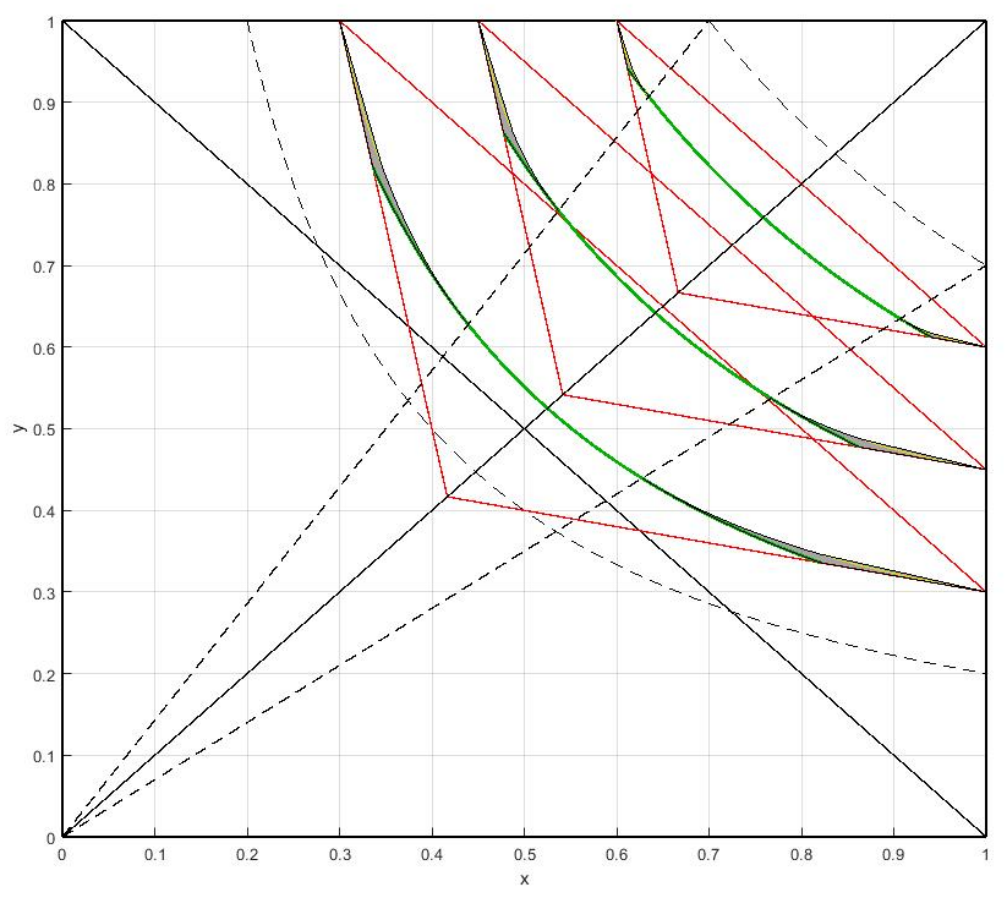

Figure 7: Cross-sections of convex hull vs. center cone only with RLT constraints

The fact that the center constraint is globally valid means that we can approximate $\operatorname{conv}\left(\mathcal{F}^{\prime}\right)$ by using this one SOC constraint together with the RLT constraints $(2 \mathrm{c})-(2 \mathrm{~d})$, where these RLT constraints can be tightened by using the values $l_{x}=l_{y}=l_{z}$ in their definitions, as in (1). We illustrate this approximation in Figure 6 for the case where $l_{z}=0.2, u_{z}=0.7$, as in Figure 5. It appears that the use of this one SOC constraint together with the RLT constraints gives a very close approximation of $\operatorname{conv}\left(\mathcal{F}^{\prime}\right)$. To show this more precisely, in Figure 7 we consider the same case of $l_{z}=0.2, u_{z}=0.7$ but show three slices, or cross-sections, corresponding to the values $z=0.3, z=0.45$ and $z=0.6$. At each value for $z$ the gray shaded area is the difference between $\operatorname{conv}\left(\mathcal{F}^{\prime}\right)$ as given by the three SOC constraints from Proposition 3 and the region determined by the center SOC constraint (6) combined with the RLT constraints (2c) (2d).

In addition to the approximation based on one SOC constraint, it is possible to give an exact disjunctive represention of $\operatorname{conv}\left(\mathcal{F}^{\prime}\right)$ over the entire region corresponding to the bounds 
$l=\left(0,0, l_{z}\right), u=\left(1,1, u_{z}\right)$ by using additional variables $\left(\lambda_{i}, x_{i}, y_{i}, z_{i}\right), i=1,2,3$, where $\lambda \geq 0, u_{z} x_{1} \leq y_{1} \leq x_{1} / u_{z}, y_{2} \leq u_{z} x_{2}, x_{3} \leq u_{z} y_{3}$, and

$$
x=\sum_{i=1}^{3} x_{i}, \quad y=\sum_{i=1}^{3} y_{i}, \quad z=\sum_{i=1}^{3} z_{i}, \quad \sum_{i=1}^{3} \lambda_{i}=1 .
$$

Each $\left(x_{i}, y_{i}, z_{i}\right)$ is then constrained to be in one of the regions given in Proposition 3 , homogenized using the variable $\lambda_{i}$. We omit the straightforward details.

\subsection{Volume computation}

As an application of the above results, in this section we will compare the volumes of $\operatorname{conv}\left(\mathcal{F}^{\prime}\right)$ that are obtained by applying the SOC constraints described in Propositions 1 and 2 to the volumes of the regions corresponding to the RLT constraints and the simple bound constraints $z \leq u_{z}$ or $z \geq l_{z}$ (but not both). Computing these volumes will also allow us to compute the total volume reduction that is obtained by creating two subproblems, one corresponding to impoing an upper bound $z \leq b$ and the other a lower bound $z \geq b$.

In the case of an upper bound $z \leq u_{z}$, it is straightforward to compute that the volume of the RLT region with the additional constraint $z \leq u_{z}$ is $u_{z}\left(u_{z}{ }^{2}-3 u_{z}+3\right) / 6$, and using a simple integration calculation, the volume removed by adding the SOC constraint in Proposition 1 is $u_{z}^{2}\left(u_{z}-1-\ln \left(u_{z}\right)\right) / 3$. The volume of $\operatorname{conv}\left(\mathcal{F}^{\prime}\right)$ with bounds $l=(0,0,0), u=\left(1,1, u_{z}\right)$ is therefore

$$
\frac{u}{6}\left(3+2 u_{z} \ln \left(u_{z}\right)-u_{z}-u_{z}^{2}\right) .
$$

In the case of a lower bound $z \geq l_{z}$, the volume of the RLT region with the added constraint $z \geq l_{z}$ is $\left(1+l_{z}\right)^{3} / 6$, where here we impose the RLT constraints (2c) - (2d) using $l_{x}=l_{y}=0$. The volume removed by adding the SOC constraint in Proposition (2) can be computed to be $l_{z}\left(1-l_{z}\right)\left(l_{z}-1-\ln \left(l_{z}\right)\right) / 3$. The volume of $\operatorname{conv}\left(\mathcal{F}^{\prime}\right)$ with bounds $l=\left(0,0, l_{z}\right), u=(1,1,1)$ is therefore

$$
\frac{1-l_{z}}{6}\left(1+2 l_{z} \ln \left(l_{z}\right)-l_{z}^{2}\right)
$$

We illustrate these volume computations in Figure 8. Let $b$ represent the bound depicted on the horizontal access. In the figure the UB: SOC+RLT series shows the volume of $\operatorname{conv}\left(\mathcal{F}^{\prime}\right)$ 


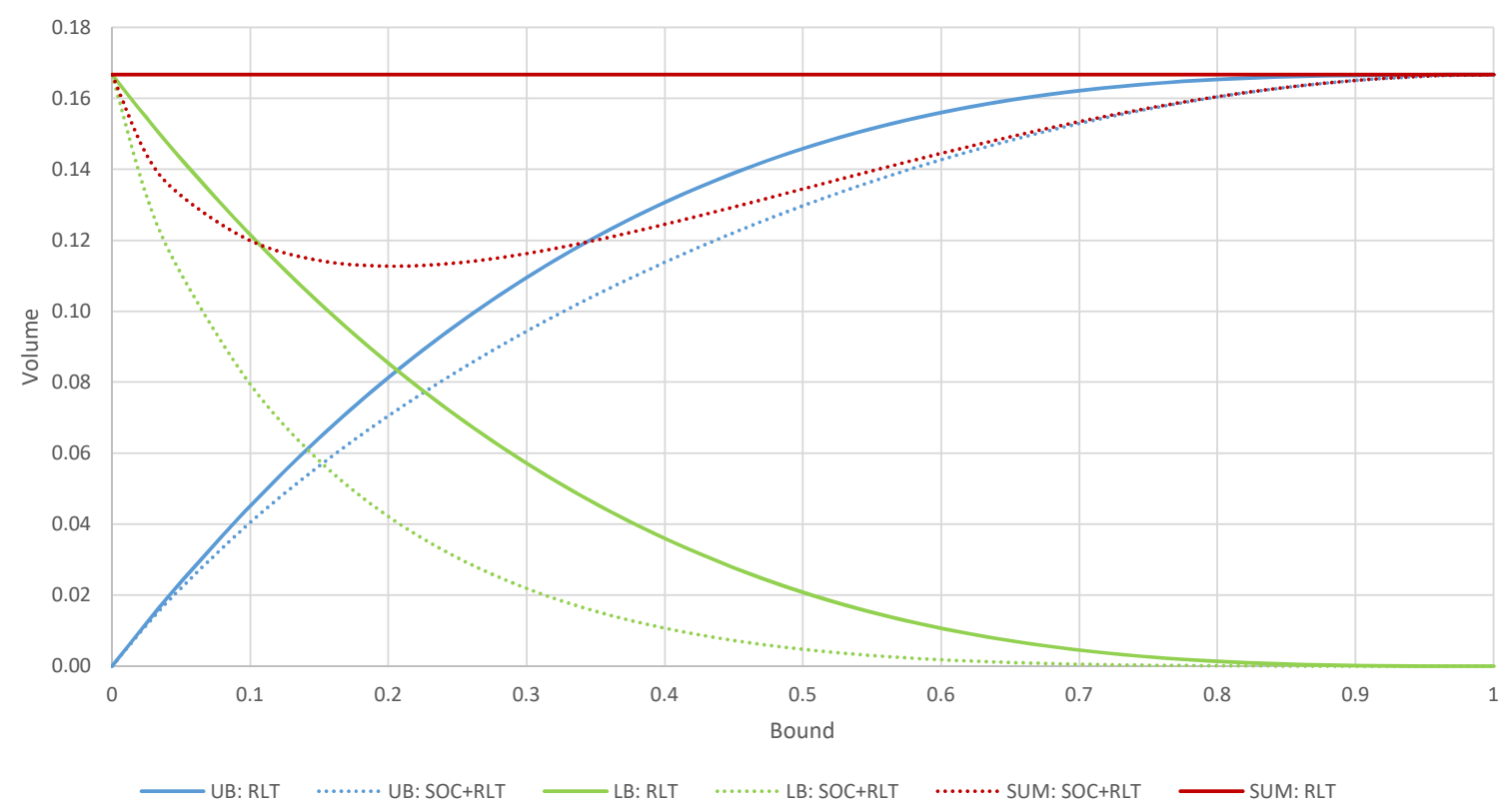

Figure 8: Volume comparisons for convex hulls versus RLT regions with added bounds on $z$.

with an upper bound $u_{z}=b$ from (7), and for comparison UB: RLT shows the volume of the RLT region cut at $z=u_{z}=b$. The LB: SOC+RLT series similarly shows the volume of $\operatorname{conv}\left(\mathcal{F}^{\prime}\right)$ with a lower bound $l_{z}=b$ from (7), and for comparison LB: RLT shows the volume of the RLT region cut at $z=l_{z}=b$. The SUM: SOC+RLT series shows the sum of the two volumes from (7) and (8) if $l_{z}=u_{z}=b$. The sum of the volumes of the two RLT regions, one cut from below at $l_{z}=b$ and the other cut from above at $u_{z}=b$, is constant and equal to $1 / 6$. From the chart it is evident that the sum of the volumes of the two convex hulls is minimized at approximately $b=0.2$; the exact minimizer satisfies the nonlinear equation $\ln (b)=2(b-1)$. In Figure 9 , we graph the ratio of the volume (7) to that of the RLT region cut at $u_{z}=b$, the ratio of the volume (8) to that of the RLT region cut at $l_{z}=b$, and the ratio of the sum of the two volumes to that of the total RLT region. The volume of the sum is reduced by approximately $32.4 \%$ at the minimizing value. This has an interesting interpretation as the possible effect of applying spatial branching to the continuous variable $z$, where one subproblem has an upper bound $u_{z}=b$ and the other has a lower bound $l_{z}=b$. In Figure 10 , we illustrate the effect of such a branching by showing the convex hulls for $u_{z}=0.3$ and $l_{z}=0.3$; in this case a total of approximately 


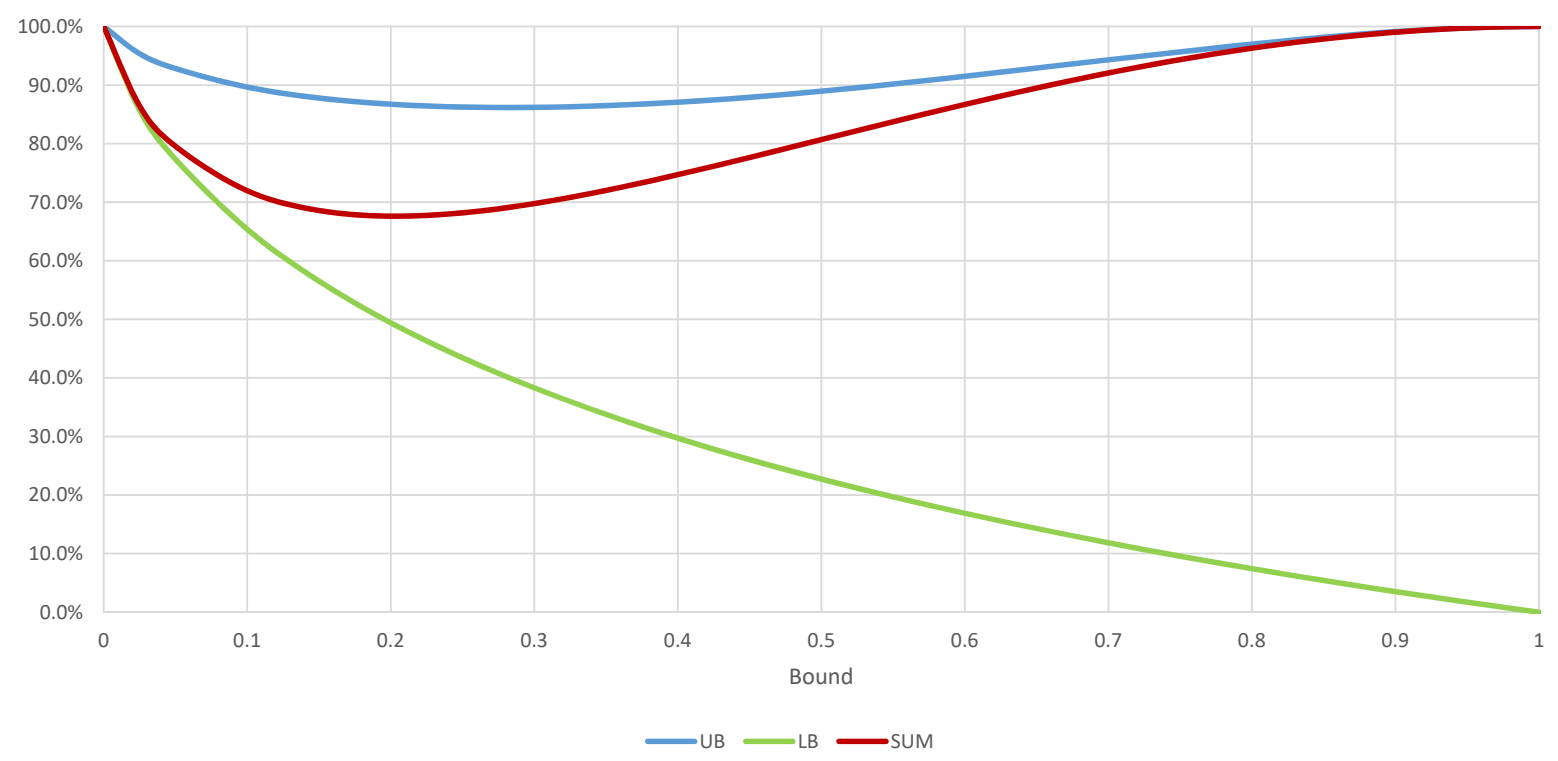

Figure 9: Volume ratios between convex hulls and RLT regions with added bounds on $z$

$30 \%$ of the volume of the original RLT region is removed by considering the two subproblems. See [9] for a recent survey of volume-based comparisons of polyhedral relxations for nonconvex optimization, and [17] for an application to branching-point selection in the presence of trilinear terms.

\section{Convex hull representation with general $\left(l_{x}, l_{y}\right)$}

In this section, we consider the case where the original variables $(x, y)$ have more general bounds of the form $l_{x} \leq x \leq u_{x}, l_{y} \leq y \leq u_{y}$. In particular, $l_{x}$ and $l_{y}$ can be positive. We continue to assume without loss of generality that $u_{x}=u_{y}=1$ since this can always be achieved by a simple rescaling of $x$ and/or $y$. Furthermore, as discussed in the introduction, we now assume without loss of generality that (1) holds.

\subsection{Non-trivial lower bound on $x y$ with general $\left(l_{x}, l_{y}\right)$}

With general lower bounds on $(x, y)$ and a non-trivial lower bound on the product $z, \operatorname{conv}\left(\mathcal{F}^{\prime}\right)$ can be described almost identically to the representation given in Proposition 2 for the case of $l_{x}=l_{y}=0$. 


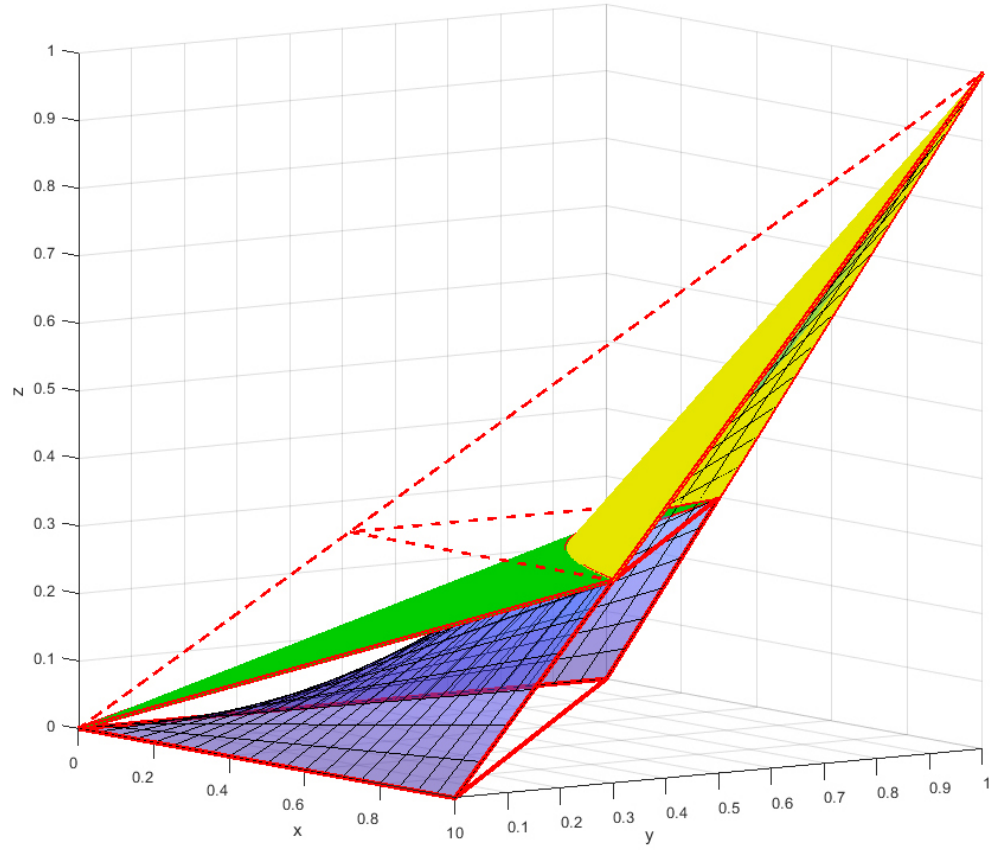

Figure 10: Effect of spatial branching on $z$

Proposition 4. Let $l=\left(l_{x}, l_{y}, l_{z}\right), u=(1,1,1)$ where $0 \leq l_{x} l_{y}<l_{z}<1$. Then $\operatorname{conv}\left(\mathcal{F}^{\prime}\right)$ is given by the RLT constraints (2), the bound $z \geq l_{z}$ and the SOC constraint $\sqrt{(\hat{x}, \hat{y}) M(\hat{x}, \hat{y})^{T}} \leq$ $x+y-2 z$ where $\hat{x}:=1-x, \hat{y}:=1-y$, and

$$
M=\left(\begin{array}{cc}
1 & 2 l_{z}-1 \\
2 l_{z}-1 & 1
\end{array}\right) \succeq 0 .
$$

Proof. The construction of the SOC constraint that implies the lifted tangent inequalities is identical to the case of $l_{x}=l_{y}=0$ considered in the proof of Proposition 2, and this SOC constraint together with the RLT constraints (2) and the bound $z \leq u_{z}$ gives $\operatorname{conv}\left(\mathcal{F}^{\prime}\right)$. However, in contrast to Proposition 2, if $l_{x}>l_{z}$ then the constraint (2c) is no longer redundant, if $l_{y}>l_{z}$ the constraint $(2 \mathrm{~d})$ is no longer redundant, and in both cases the constraint $(2 \mathrm{~b})$ is no longer redundant.

In Figure 11, we illustrate $\operatorname{conv}\left(\mathcal{F}^{\prime}\right)$ for $l=(0.5,0.3,0.3), u=(1,1,1)$. Since $l_{x}>l_{z}$, the constraints (2c) and (2b) are now active. 


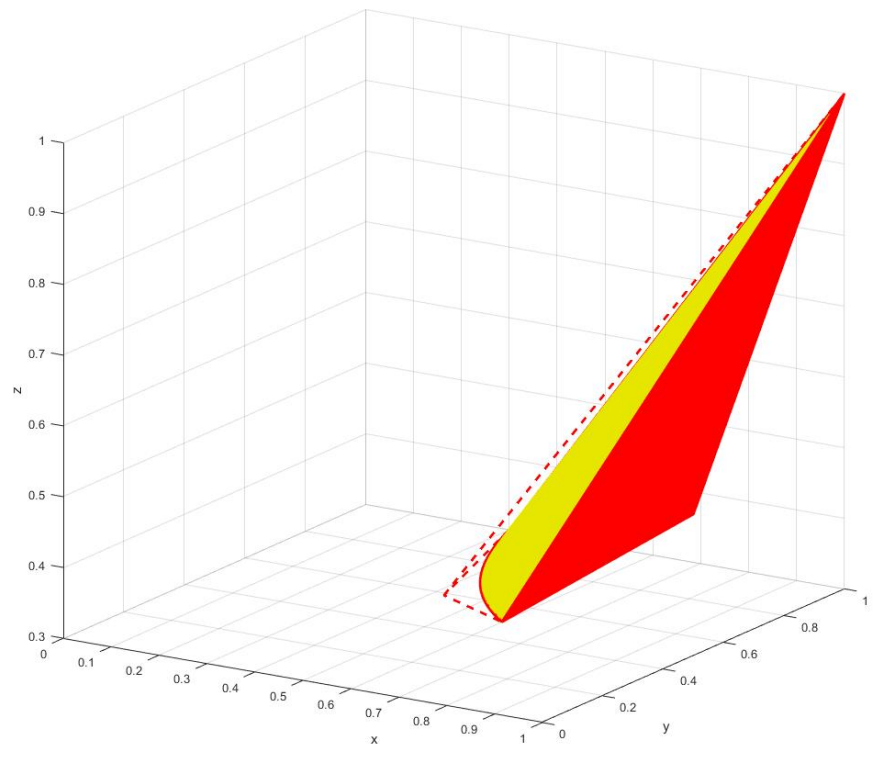

Figure 11: Convex hull with general lower bounds on $(x, y, z)$.

\subsection{Non-trivial upper bound on $x y$ with general $\left(l_{x}, l_{y}\right)$}

With general lower bounds $l_{x}$ and $l_{y}$ and a non-trivial upper bound on $z$, the geometry of $\operatorname{conv}\left(\mathcal{F}^{\prime}\right)$ is similar to the case of $l_{x}=l_{y}=0$ considered in Section 3.1, but the derivation of the conic constraint in SOC form is more complex. Lifted tangent inequalities now correspond to line segments joining a point $\left(\bar{x}, \bar{y}, u_{z}\right) \in \mathcal{F}^{\prime}$ with the point $\left(l_{x}, l_{y}, l_{x} l_{y}\right)$. For a point $(x, y, z)$ on such a line segment we have $x=\alpha l_{x}+(1-\alpha) \bar{x}, y=\alpha l_{y}+(1-\alpha) \bar{y}$. Writing $(\bar{x}, \bar{y})$ in terms of $(x, y)$ then results in a quadratic equation for $\alpha$, and for the appropriate root of this equation the constraint $x \leq \alpha\left(l_{x} l_{y}\right)+(1-\alpha) u_{z}$ results in the constraint

$$
\left(z-l_{y} x\right)\left(z-l_{x} y\right) \leq u_{z}\left(x-l_{x}\right)\left(y-l_{y}\right)
$$

This constraint is certainly valid for all $(x, y, z) \in \operatorname{conv}\left(\mathcal{F}^{\prime}\right)$. In particular, if $z=x y \leq u_{z}$ then $\left(z-l_{y} x\right)=x\left(y-l_{y}\right)$ and $\left(z-l_{x} y\right)=y\left(x-l_{x}\right)$, so $\left(z-l_{y} x\right)\left(z-l_{x} y\right)=x y\left(x-l_{x}\right)\left(y-l_{y}\right) \leq$ $u_{z}\left(x-l_{x}\right)\left(y-l_{y}\right)$. Note that if $l_{x}=l_{y}=0$, then (9) is exactly the SOC constraint $z^{2} \leq u_{z} x y$ from Proposition 1. If either $l_{x}=0$ or $l_{y}=0$ it is also easy to put the constraint (9) into the form of an SOC constraint, but when $l_{x}>0, l_{y}>0$ this is nontrivial. 
Proposition 5. Let $l=\left(l_{x}, l_{y}, 0\right), u=\left(1,1, u_{z}\right)$ where $0<u_{z}<1$. Then $\operatorname{conv}\left(\mathcal{F}^{\prime}\right)$ is given by the RLT constraints (2), the bound $z \leq u_{z}$ and the SOC constraint

$$
u_{z}\left(z-l_{x} l_{y}\right)^{2} \leq\left(u_{z}\left(x-l_{x}\right)+l_{x}\left(z-l_{y} x\right)\right)\left(u_{z}\left(y-l_{y}\right)+l_{y}\left(z-l_{x} y\right)\right) .
$$

Proof. The convex hull of $\mathcal{F}^{\prime}$ is given by the RLT constraints, the bound $z \leq u_{z}$, and the lifted tangent inequalities, and the latter are implied by the constraint (9). By a direct computation the constraint (10) is equivalent to multiplying both sides of (9) by the constant $u_{z}-l_{x} l_{y}>0$. Moreover, $x \geq l_{x}, y \geq l_{y}$ and the RLT constraint (2b) together imply that $z \geq l_{y} x$ and $z \geq l_{x} y$. Both terms that form the product on the right-hand side of 10 can therefore be assumed to be nonnegative, so (10) is an SOC constraint that implies the lifted tangent inequalities.

The proof of Proposition 5 requires only that $(9)$ and (10) are equivalent, but it is worth noting how (10) was obtained. This was accomplished by writing (9) in the form $v^{T} Q v \leq 0$, where $v=(1, x, y, z)^{T}$, and then performing symbolic, symmetric transformations on $Q$ so as to obtain

$$
S Q S^{T}=\hat{Q}=\left(\begin{array}{cccc}
2 u_{z} & 0 & 0 & 0 \\
0 & 0 & -1 & 0 \\
0 & -1 & 0 & 0 \\
0 & 0 & 0 & 0
\end{array}\right) .
$$

Note that $v^{T} Q v=v^{T} S^{-1} \hat{Q} S^{-T} v$, and $\hat{Q}$ has exactly one negative eigenvalue. The spectral decomposition of $\hat{Q}$ and the symbolic matrix $S^{-T}$ were together used to obtain the equivalent SOC constraint (10). In Figure 12, we illustrate $\operatorname{conv}\left(\mathcal{F}^{\prime}\right)$ for the case with $u_{z}=0.7$ and lower bounds $l_{x}=0.4, l_{y}=0.5$.

\subsection{Non-trivial lower and upper bounds on $x y$ with general $\left(l_{x}, l_{y}\right)$}

We now consider the most general case for $\mathcal{F}^{\prime}$, where $l=\left(l_{x}, l_{y}, l_{z}\right)>0$ and $u_{z}<1$. We continue to assume that $u_{x}=u_{y}=1$, and $l_{z} \leq l_{x} \leq u_{z}, l_{z} \leq l_{y} \leq u_{z}$ as described at the beginning of the section. Finally we assume that $l_{x} l_{y}<l_{z}$, since otherwise $l_{x} l_{y} \geq l_{z}$ implies that the lower bound $x y \geq l_{z}$ is redundant, which is the case of the previous section.

In order to describe the possible representations for $\operatorname{conv}\left(\mathcal{F}^{\prime}\right)$, it is very convenient to dissect the domain for possible values of $\left(l_{x}, l_{y}\right)$ into regions where representations of a particular type 


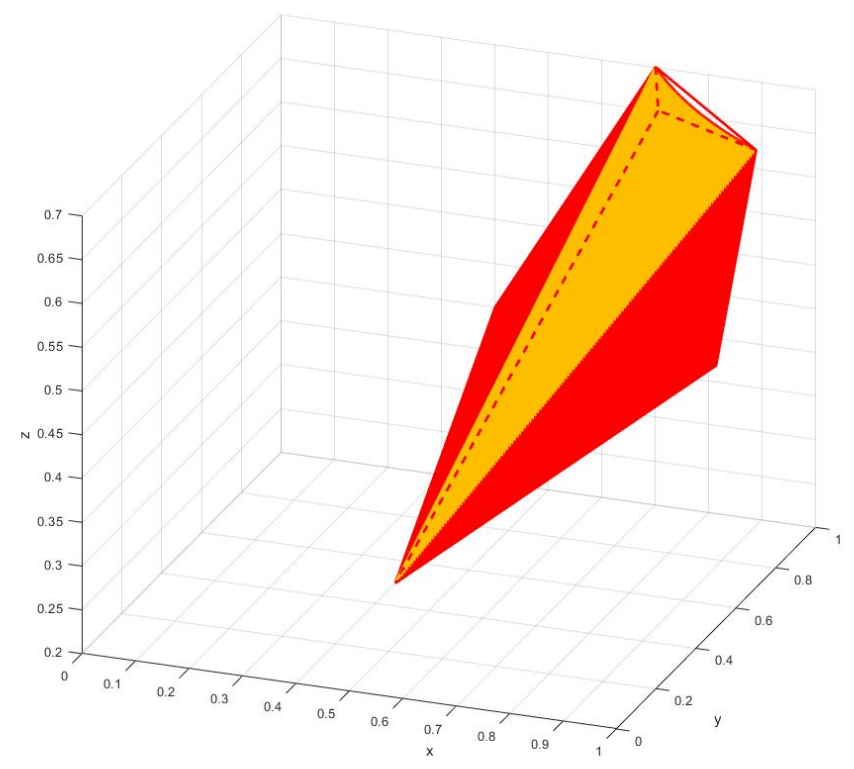

Figure 12: Convex hull with general lower bounds on $(x, y)$ and non-trivial upper bound on $z$

occur. These regions naturally involve the values $\sqrt{l_{z} u_{z}}$ and $\sqrt{l_{z} / u_{z}}$. In particular, note that the point $(x, y)=\left(\sqrt{l_{z} / u_{z}}, \sqrt{l_{z} u_{z}}\right)$ is the intersection of the line $y=u_{z} x$ and the curve $x y=l_{z}$, while $(x, y)=\left(\sqrt{l_{z} u_{z}}, \sqrt{l_{z} / u_{z}}\right)$ is the intersection of the line $x=u_{z} y$ and the curve $x y=l_{z}$. Under our assumptions for the values of $l$ and $u$, the possible regions for $\left(l_{x}, l_{y}\right)$ are as follows and are illustrated in Figure 13 for the case of $l_{z}=0.1, u_{z}=0.7$.
A. $l_{x} \geq \sqrt{l_{z} u_{z}}, l_{y} \geq \sqrt{l_{z} u_{z}}, l_{x} l_{y}<l_{z}$.
B. $l_{z} \leq l_{x} \leq \sqrt{l_{z} u_{z}}, l_{z} \leq l_{y} \leq \sqrt{l_{z} u_{z}}$.
C. $l_{z} \leq l_{x} \leq \sqrt{l_{z} u_{z}}, \sqrt{l_{z} u_{z}} \leq l_{y} \leq \sqrt{l_{z} / u_{z}}$.
D. $l_{x} \geq l_{z}, \sqrt{l_{z} / u_{z}} \leq l_{y} \leq u_{z}, l_{x} l_{y} \leq l_{z}$.
E. $\sqrt{l_{z} u_{z}} \leq l_{x} \leq \sqrt{l_{z} / u_{z}}, l_{z} \leq l_{y} \leq \sqrt{l_{z} u_{z}}$.
F. $\sqrt{l_{z} / u_{z}} \leq l_{x} \leq u_{z}, l_{y} \geq l_{z}, l_{x} l_{y} \leq l_{z}$.

It is clear that regions $\mathrm{E}$ and $\mathrm{F}$ correspond to regions $\mathrm{C}$ and $\mathrm{D}$, respectively, with the roles of $x$ and $y$ interchanged. Since we can assume without loss of generality that $l_{x} \leq l_{y}$, in the results below we will only consider regions A-D. We omit proofs of these results since in all 


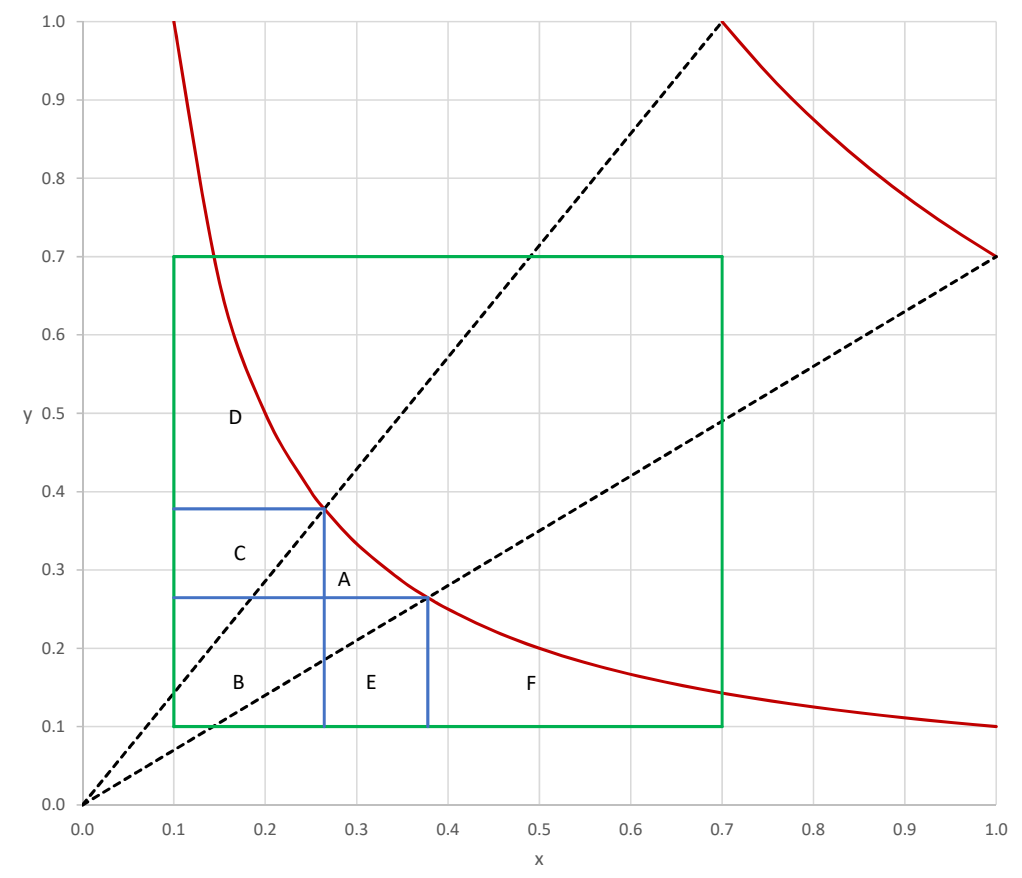

Figure 13: Domains for $\left(l_{x}, l_{y}\right)$ with $l_{z}=0.1, u_{z}=0.7$

cases they are based on SOC representations for lifted tangent inequalities described in earlier sections. In each of the four cases, the representation of $\operatorname{conv}\left(\mathcal{F}^{\prime}\right)$ will include several SOC constraints that imply the lifted tangent inequalities on different $(x, y)$ domains. In Figure 14 , we illustrate these domains using values of $\left(l_{x}, l_{y}\right)$ corresponding to each of the regions A-D, with $l_{z}=0.1, u_{z}=0.7$ as in Figure 13 . In the figure, the boundaries of domains on which different SOC constraints imply the lifted tangent inequalities are given by solid black lines, and blue lines indicate the region $(x, y) \geq\left(l_{x}, l_{y}\right)$.

Proposition 6. Suppose that $\left(l_{x}, l_{y}\right)$ is in region $A$. Then $\operatorname{conv}\left(\mathcal{F}^{\prime}\right)$ is given by the the $R L T$ constraints, the bounds $l_{z} \leq z \leq u_{z}$, and the following three SOC constraints, each applicable in a different region:

1. The constraint (6), applicable if $y \geq\left(l_{y}{ }^{2} / l_{z}\right) x, y \leq\left(l_{z} / l_{x}{ }^{2}\right) x$.

2. The constraint (10), but with $l_{x}$ replaced by $l_{z} / l_{y}$, applicable if $y \leq\left(l_{y}{ }^{2} / l_{z}\right) x$.

3. The constraint (10), but with $l_{y}$ replaced by $l_{z} / l_{x}$, applicable if $y \geq\left(l_{z} / l_{x}^{2}\right) x$.

Note that the first constraint in Proposition 6 is exactly the constraint based on $\left(l_{z}, u_{z}\right)$ from 

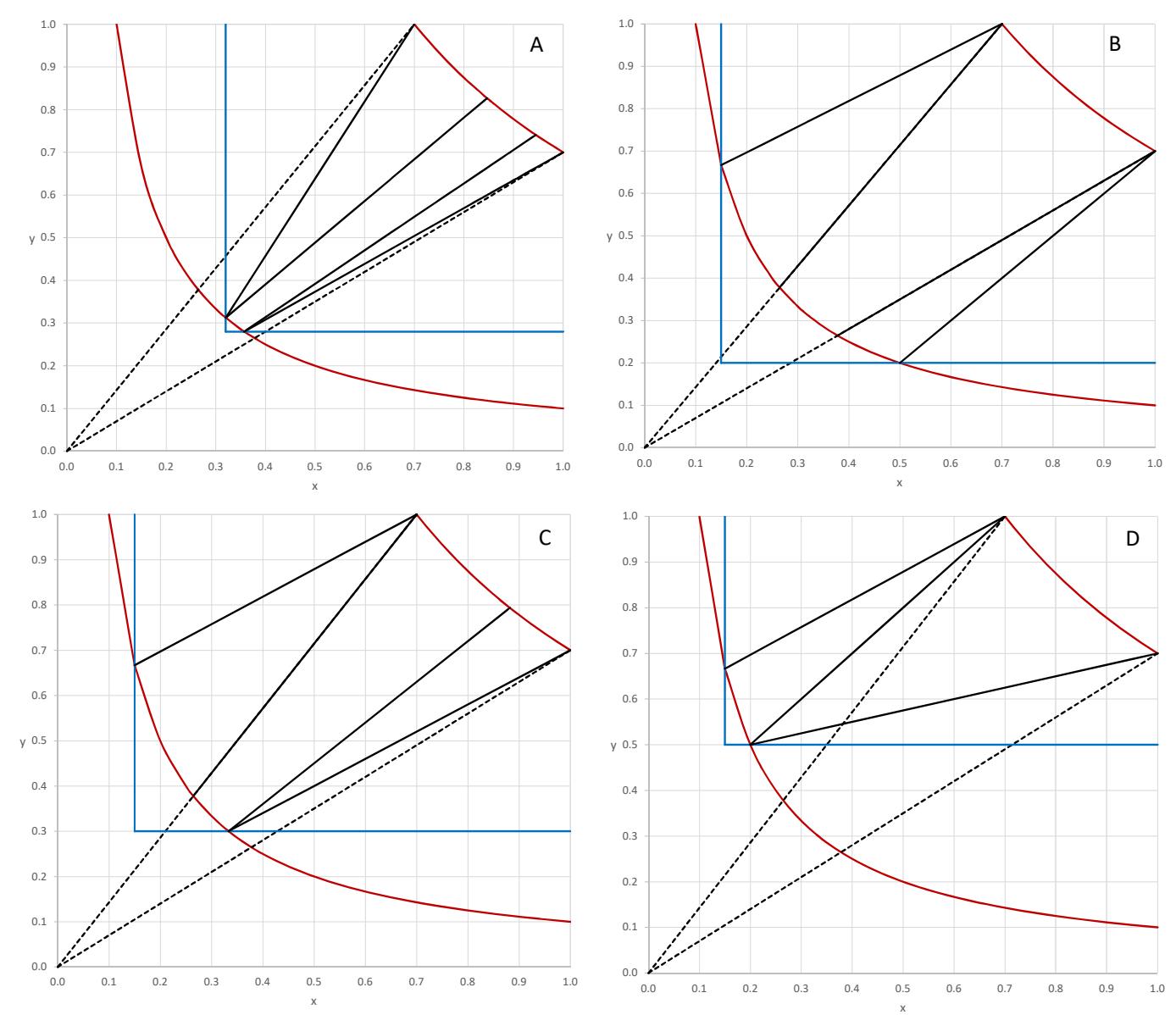

Figure 14: Domains for SOC constraints with $l_{z}=0.1, u_{z}=0.7$

Proposition 3 . This constraint is globally valid and is binding in the region $y \geq\left(l_{y}{ }^{2} / l_{z}\right) x, y \leq$ $\left(l_{z} / l_{x}^{2}\right) x$. The second constraint corresponds to using the lower bounds $\left(l_{z} / l_{y}, l_{y}\right)$ in Proposition 5. and is certainly then valid for all $(x, y) \geq\left(l_{z} / l_{y}, l_{y}\right)$, where $l_{z} / l_{y}>l_{x}$ by assumption. Note also that $y \leq\left(l_{y}{ }^{2} / l_{z}\right) x$ and $y \geq l_{y}$ together imply that $x \geq l_{z} / l_{y}$. Similarly the third constraint is valid for all $(x, y) \geq\left(l_{x}, l_{z} / l_{x}\right)$. The regions on which the second and third constraints are actually binding can easily be determined from the points $\left(l_{z} / l_{y}, l_{y}\right),\left(l_{x}, l_{z} / l_{x}\right),\left(1, u_{z}\right)$ and $\left(u_{z}, 1\right)$; see Figure 14

For $\left(l_{x}, l_{y}\right)$ in region $\mathrm{B}$, the representation with lower bounds $\left(l_{x}, l_{y}\right)>0$ is essentially identical to that given in Proposition 3 , except that the RLT constraints can now all be active.

Proposition 7. Suppose that $\left(l_{x}, l_{y}\right)$ is in region B. Then $\operatorname{conv}\left(\mathcal{F}^{\prime}\right)$ is given by the RLT con- 
straints, the bounds $l_{z} \leq z \leq u_{z}$, and the three SOC constraints from Proposition 3 where each constraint is applicable for the $(x, y)$ values as given in Proposition 3

For $\left(l_{x}, l_{y}\right)$ in region $\mathrm{C}$, the representation with lower bounds $\left(l_{x}, l_{y}\right)>0$ uses a mixture of the SOC constraints that appear in Propositions 6 and 7

Proposition 8. Suppose that $\left(l_{x}, l_{y}\right)$ is in region $C$. Then $\operatorname{conv}\left(\mathcal{F}^{\prime}\right)$ is given by the RLT constraints, the bounds $l_{z} \leq z \leq u_{z}$, and the following three SOC constraints, each applicable on a different region:

1. The constraint $\left(z+\sqrt{l_{z} u_{z}}\right)^{2} \leq\left(\sqrt{l_{z}}+\sqrt{u_{z}}\right)^{2} x y$, applicable if $y \geq\left(l_{y}^{2} / l_{z}\right) x, y \leq x / u_{z}$.

2. The constraint (10), but with $l_{x}$ replaced by $l_{z} / l_{y}$, applicable if $y \leq\left(l_{y}^{2} / l_{z}\right) x$.

3. The constraint $\sqrt{(\hat{x}, \hat{y}) M_{2}(\hat{x}, \hat{y})^{T}} \leq x+u_{z} y-2 z$, where $\hat{x}:=u_{z}-x, \hat{y}:=1-y$ and $M_{2} \succeq 0$ is given in (5), applicable if $x \leq u_{z} y$.

The remaining case, where $\left(l_{x}, l_{y}\right)$ is in region $\mathrm{D}$, is qualitatively different from the three previous cases because the "center cone" from Proposition 3 does not appear in the representation of $\operatorname{conv}\left(\mathcal{F}^{\prime}\right)$. There are only two SOC cones in the representation, and the boundary between the regions on which these cones are active is not homogeneous. This boundary is given by the line which joins the points $\left(l_{z} / l_{y}, l_{y}\right)$ and $\left(u_{z}, 1\right)$, whose equation is $y=a+b x$, where

$$
a=\frac{u_{z} l_{y}^{2}-l_{z}}{u_{z} l_{y}-l_{z}}, \quad b=\frac{l_{y}\left(1-l_{y}\right)}{u_{z} l_{y}-l_{z}} .
$$

Proposition 9. Suppose that $\left(l_{x}, l_{y}\right)$ is in region $D$. Then $\operatorname{conv}\left(\mathcal{F}^{\prime}\right)$ is given by the the $R L T$ constraints, the bounds $l_{z} \leq z \leq u_{z}$, and the following two SOC constraints, each applicable in a different region:

1. The constraint (10), but with $l_{x}$ replaced by $l_{z} / l_{y}$, applicable in the region $y \leq a+b x$, where $a$ and b are given by (11).

2. The constraint $\sqrt{(\hat{x}, \hat{y}) M_{2}(\hat{x}, \hat{y})^{T}} \leq x+u_{z} y-2 z$, where $\hat{x}:=\left(u_{z}-x\right), \hat{y}:=(1-y)$ and $M_{2} \succeq 0$ is given in (5), applicable if $y \geq a+b x$, where $a$ and $b$ are given by (11). 


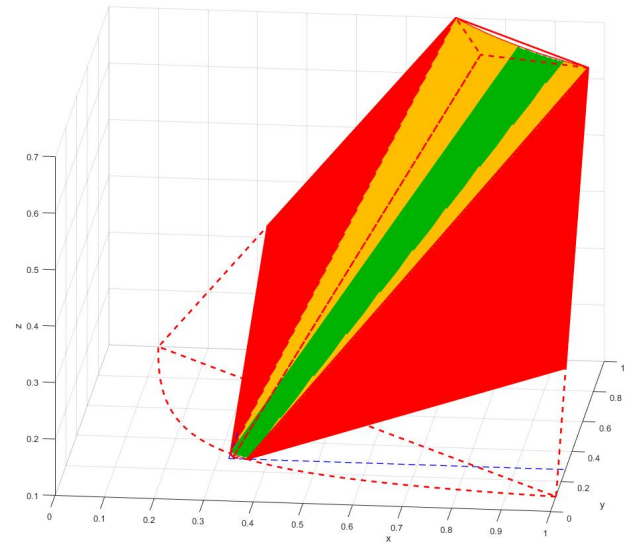

(a) Case A: $l_{x}=0.32, l_{y}=0.28$

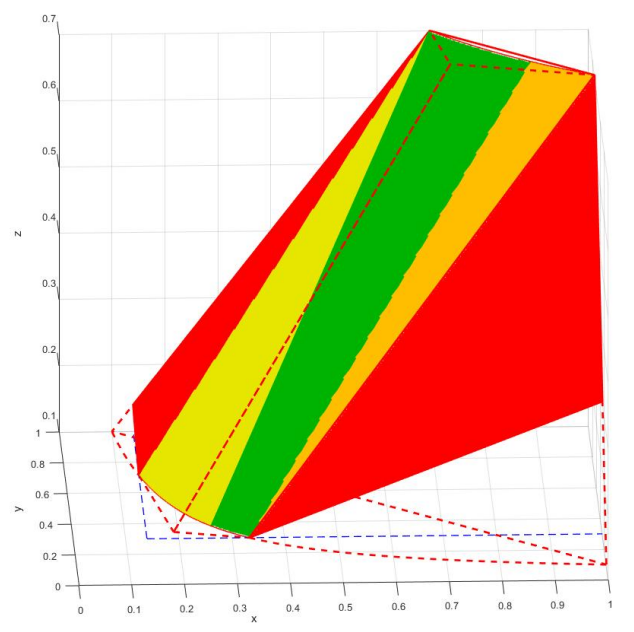

(c) Case C: $l_{x}=0.14, l_{y}=0.3$

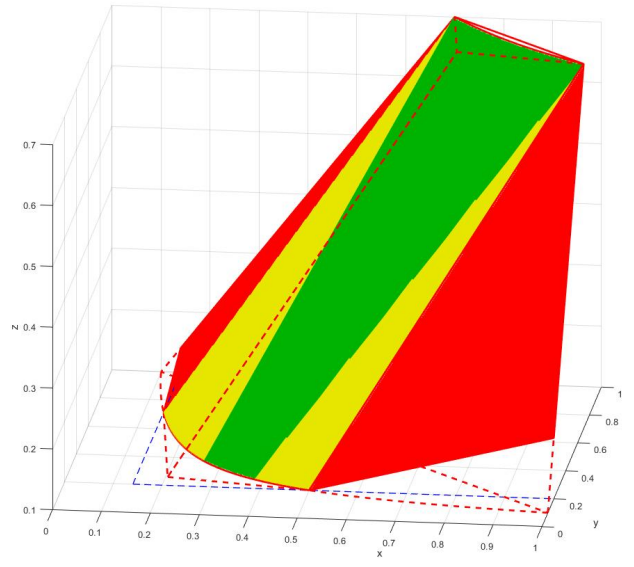

(b) Case B: $l_{x}=0.14, l_{y}=0.2$

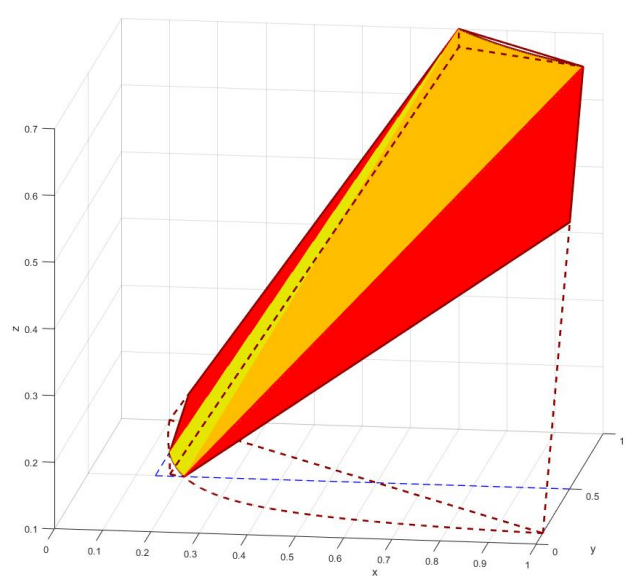

(d) Case D: $l_{x}=0.14, l_{y}=0.5$

Figure 15: Convex hulls with general lower bounds on $x$ and $y: l_{z}=0.1, u_{z}=0.7$ 
In Figure 15, we illustrate examples of $\operatorname{conv}\left(\mathcal{F}^{\prime}\right)$ for $l_{z}=0.1, u_{z}=0.7$ and values of $l_{x}, l_{y}$ in each of the four regions A-D shown in Figure 13 .

Note that neither of the constraints in Proposition 9 is globally valid; the first is valid for $x \geq l_{z} / l_{y}$ and the second is valid for $y \geq x / u_{z}$. In fact, all of the representations in this section involve some SOC constraints that are not globally valid. In order to represent $\operatorname{conv}\left(\mathcal{F}^{\prime}\right)$ over the entire set of feasible $(x, y)$ in any of these cases, one could use a disjunctive representation as described at the end of Section 3.3. Alternatively, we could always use the SOC constraint (6) together with the constraint (10) since both of these are globally valid. We would expect that these two SOC constraints together with the RLT constraints and bounds on $z$ would give a close approximation of $\operatorname{conv}\left(\mathcal{F}^{\prime}\right)$ in many cases.

\section{Conclusion}

We have shown that in all cases, $\operatorname{conv}\left(\mathcal{F}^{\prime}\right)$ can be represented using a combination of RLT constraints, bound(s) on the product variable $z$ and no more than three SOC constraints. In cases where more than one SOC constraint is required to represent $\operatorname{conv}\left(\mathcal{F}^{\prime}\right)$, each such constraint is applicable on a subset of the domain of $(x, y)$ values, but one or two globally valid SOC constraints can be used together with the RLT constraints and bounds on $z$ to approximate $\operatorname{conv}\left(\mathcal{F}^{\prime}\right)$.

Our results suggest a number of promising directions for future reserach. First, it may be possible to extend some of these results to the case of multilinear terms, where $z$ is the product of $n>2$ variables; an extension of the lifted tangent inequalities to $n>2$ is described in [5]. Second, it would be interesting to extend the convex hull description for the complete 5-variable system in [3] to allow for bounds on the product $x y$. Note that the the results of [3] already apply to arbitrary bounds $0 \leq l_{x} \leq x \leq u_{x}, 0 \leq l_{y} \leq y \leq u_{y}$, and bounds on the squared terms $x^{2}$ and/or $y^{2}$ are equivalent to bounds on the original variables $(x, y)$. However, the results of [3] do not allow for an additional bound on the product $x y$. Finally, an extension of the results here to the case of $z=x^{T} y$, where $x \in \mathbb{R}_{+}^{n}, y \in \mathbb{R}_{+}^{n}$, would be very significant since such bilinear terms appear in many applications. 


\section{Acknowledgement}

Kurt Anstreicher would like to thank Pietro Belotti, Jeff Linderoth and Mohit Tawarmalani for very helpful conversations on the topic of this paper.

\section{References}

[1] F. A. Al-Khayyal and J. E. Falk. Jointly constrained biconvex programming. Math. Oper. Res., 8(2):273-286, 1983.

[2] K. M. Anstreicher. Semidefinite programming versus the reformulation-linearization technique for nonconvex quadratically constrained quadratic programming. J. Global Optim., 43(2-3):471-484, 2009.

[3] K. M. Anstreicher and S. Burer. Computable representations for convex hulls of lowdimensional quadratic forms. Math. Program., 124(1-2):33-43, 2010.

[4] P. Belotti, J. Lee, L. Liberti, F. Margot, and A. Wächter. Branching and bounds tightening techniques for non-convex minlp. Optim. Methods \& Software, 24(4-5):597-634, 2009.

[5] P. Belotti, A. J. Miller, and M. Namazifar. Valid inequalities and convex hulls for multilinear functions. Electronic Notes in Discrete Mathematics, 36:805-812, 2010.

[6] S. Boyd and L. Vandenberghe. Convex Optimization. Cambridge University Press, 2004.

[7] S. S. Dey, A. Santana, and Y. Wang. New SOCP relaxation and branching rule for bipartite bilinear programs. Optim. Eng., 20(2):307-336, 2019.

[8] M. Jach, D. Michaels, and R. Weismantel. The convex envelope of $(n-1)$-convex functions. SIAM. J. Optim., 19:1451-1466, 2008.

[9] J. Lee, D. Skipper, and E. Speakman. Algorithmic and modeling insights via volumetric comparison of polyhedral relaxations. Math. Program. B, 170:121-140, 2018. 
[10] J. Linderoth. A simplicial branch-and-bound algorithm for solving quadratically constrained quadratic programs. Math. Program., 103(2):251-282, 2005.

[11] A. J. Miller, P. Belotti, and M. Namazifar. Linear inequalities for bounded products of variables. SIAG/OPT Views and News, 22(1):1-8, 2011.

[12] T. T. Nguyen, J.-P. P. Richard, and M. Tawarmalani. Deriving convex hulls through lifting and projection. Math. Program., 169(2):377-415, 2018.

[13] N. Sahinidis. BARON: a general purpose global optimization software package. J. Global Optim., 8:201-205, 1996.

[14] A. Santana and S. S. Dey. The convex hull of a quadratic constraint over a polytope. Manuscript, Georgia Institute of Technology, Atlanta, GA, July 2019. http://www . optimization-online.org/DB_HTML/2018/12/7004.html.

[15] H. D. Sherali and W. P. Adams. A Reformulation-Linearization Technique for Solving Discrete and Continuous Nonconvex Problems. Springer, 2013.

[16] H. D. Sherali and A. Alameddine. A new reformulation-linearization technique for bilinear programming problems. J. Global Opt., 2(4):379-410, 1992.

[17] E. Speakman and J. Lee. On branching-point selection for trilinear monomials in spatial branch-and-bound: the hull relaxation. J. Global Optim., 72(2):129-153, 2018. 